\title{
WestVirginiaUniversity
}

THE RESEARCH REPOSITORY @ WVU

Graduate Theses, Dissertations, and Problem Reports

2008

\section{Molecular prediction of drug response using machine learning methods}

Zhenyu Ding

West Virginia University

Follow this and additional works at: https://researchrepository.wvu.edu/etd

\section{Recommended Citation}

Ding, Zhenyu, "Molecular prediction of drug response using machine learning methods" (2008). Graduate Theses, Dissertations, and Problem Reports. 4365.

https://researchrepository.wvu.edu/etd/4365

This Thesis is protected by copyright and/or related rights. It has been brought to you by the The Research Repository @ WVU with permission from the rights-holder(s). You are free to use this Thesis in any way that is permitted by the copyright and related rights legislation that applies to your use. For other uses you must obtain permission from the rights-holder(s) directly, unless additional rights are indicated by a Creative Commons license in the record and/ or on the work itself. This Thesis has been accepted for inclusion in WVU Graduate Theses, Dissertations, and Problem Reports collection by an authorized administrator of The Research Repository @ WVU. For more information, please contact researchrepository@mail.wvu.edu. 


\title{
Molecular Prediction of Drug Response Using Machine
}

\section{Learning Methods}

\author{
Zhenyu Ding \\ Thesis Submitted to the \\ College of Engineering and Mineral Resources \\ At West Virginia University \\ In partial fulfillment of the requirements \\ For the degree of \\ Master of Science \\ In \\ Computer Science \\ Committee: \\ Nancy Lan Guo, Ph.D., Chair \\ Bojan Cukic, Ph.D. \\ Arun Ross, Ph.D.
}

Lane Department of Computer Science and Electrical Engineering

$$
\begin{gathered}
\text { Morgantown, WV } \\
2008
\end{gathered}
$$

Keywords: Machine Learning, Feature Selection, Classification, Molecular

Prediction, Chemosensitivity, Drug Response, Cancer Drug, Proteomic

Profiling, Genomic Profiling 


\section{ABSTRACT \\ Molecular Prediction of Drug Response Using Machine Learning Methods}

\section{Zhenyu Ding}

Increasing the efficiency and effectiveness of chemotherapy will rely on the ability to accurately predict an individual cancer patient's chemosensitivity to certain drugs. One approach to this issue has focused on genomic and proteomic profiling. While previous work has focused on genomic profiling, few studies have explored the correlation of proteomic profiles with drug sensitivity. Meanwhile, a novel algorithm is needed to integrate both protein and gene expression, which is important to systematically understand fundamental chemosensitivity mechanisms. In this study, we sought to explore whether proteomic signatures of untreated cancer cell lines could accurately predict their chemosensitivity. Furthermore, we developed an algorithm to integrate proteomic and genomic profiles and used them to classify the chemosensitivity of the cell lines in an attempt to determine whether the integrated profiles could further increase the accuracy of chemosensitivity prediction.

First, in order to explore whether the proteomic signatures could accurately predict chemosensitivity, we developed a machine learning model exclusively based on proteomic profiling to predict drug response. We used data from studies in which the expression levels of 52 proteins in 60 human cancer cell (NCI-60) lines were determined. The model combined random forests, Relief, and nearest neighbor algorithms to construct chemosensitivity classifiers for each cell line against each of 118 chemotherapeutic agents. The chemosensitivity prediction accuracy of all the evaluated 118 agents was significantly $(P<0.02)$ higher than random prediction accuracy. These results indicate that it is feasible to accurately predict chemosensitivity by proteomic approaches.

Next, we integrated genomic profiling into our proteomic model and developed a novel feature selection scheme to identify biomarkers from the integrated profiles in NCI-60 cell lines. Then, we used the random forests algorithm to construct chemosensitivity classifiers for the same 118 agents. Seventy-six out of the 118 classifiers could significantly $(P<0.05)$ improve the chemosensitivity prediction accuracy acquired by protein expression-based classifiers alone. These results demonstrate that our integrated genomic and proteomic approach could further increase chemosensitivity prediction accuracy.

Overall, we found that it is feasible to use proteomic signatures alone to accurately predict chemosensitivity. Integrating genomic and proteomic signatures further increases chemosensitivity prediction accuracy. 


\section{Acknowledgements}

First and foremost, I would like to express my deepest gratitude to my committee chair, Dr. Nancy Lan Guo, who answered all questions and guided me down the right path. Her wisdom and experience helped every step along the way. This project was supported by: NIH/National Center for Research Resources grant P20RR16440-03.

I am very grateful to Dr. Bojan Cukic. Thanks for his kind and warm help during my study.

I want to express my sincerest appreciation to Dr. Ross for his patience and dedication, for the valuable time that he has spent in teaching me the courses of pattern classification and helping me in this project.

I really appreciate Dr. Yan Ma. She taught me a lot of statistical skill, which helped me to finish this study. Thanks for her patience and time.

I also appreciate Drs. Zhibao Mi, Nan Song, and Janice Sabatine’s help for their advice of revising this thesis.

Finally, I would thank my family and friends. Without your love and support, I couldn't go so far. 


\section{Contents}

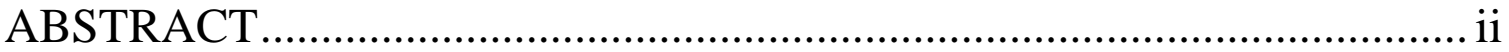

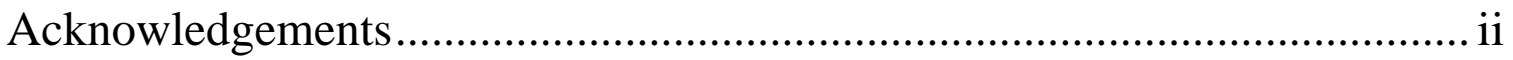

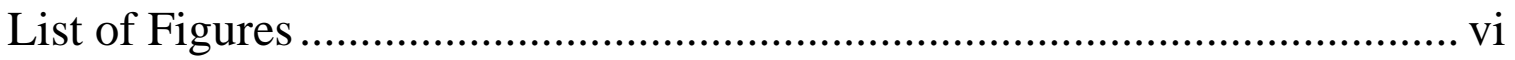

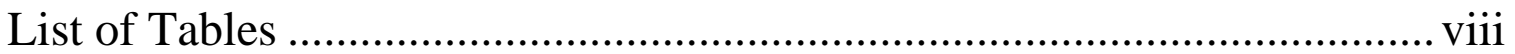

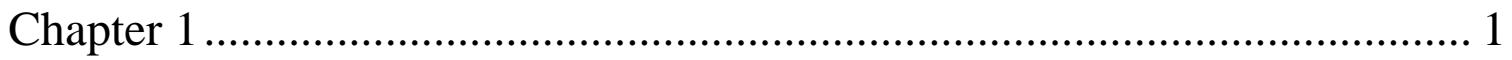

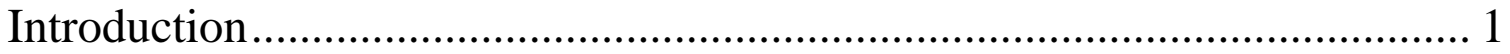

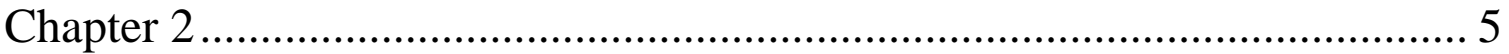

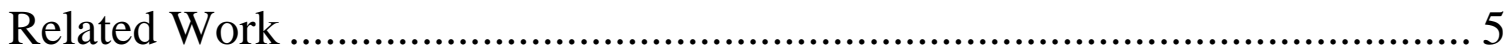

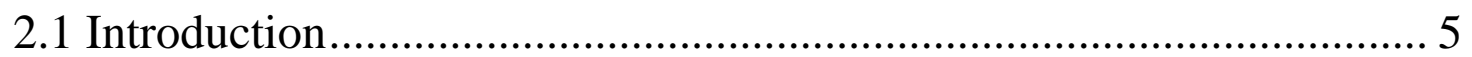

2.2 Machine Learning Algorithms......................................................... 5

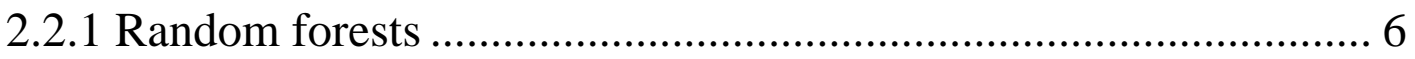

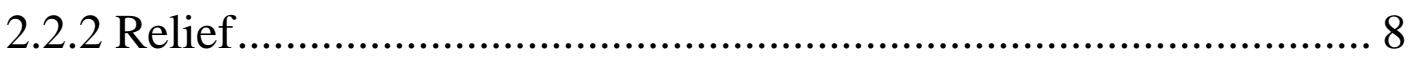

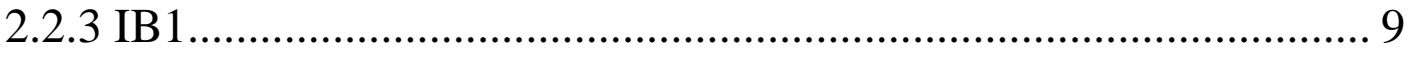

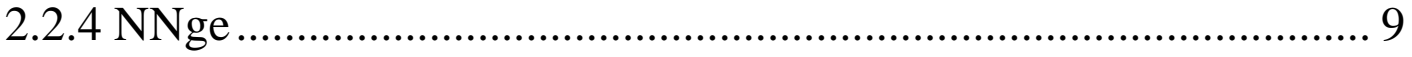

2.3 Molecular Profiling and Bioinformatics ............................................. 10

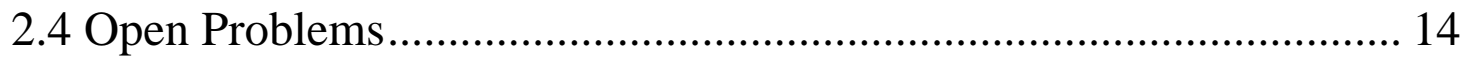

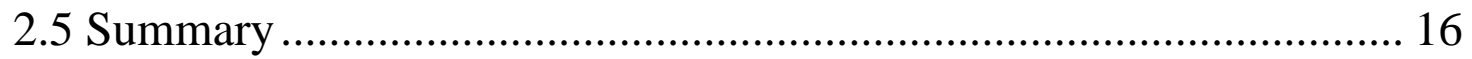

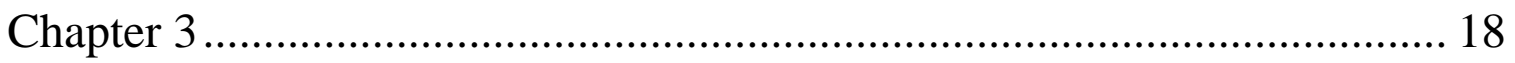

Predicting Cancer Drug Response by Proteomic Profiling .......................... 18

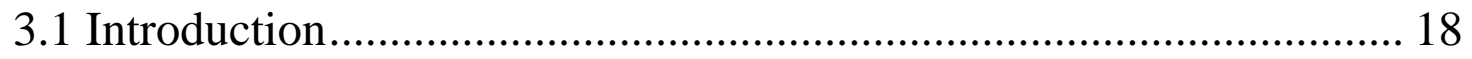

3.2 Experimental Design..................................................................... 19

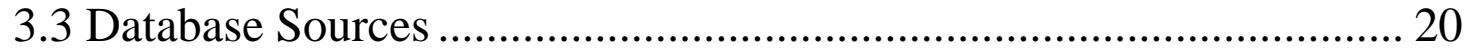

3.4 Descriptions of the Experiment ........................................................ 22

3.4.1 Defining Cutoffs of Drug Sensitivity and Resistance ................. 22

3.4.2 Selecting Protein Predictor .......................................................... 26 
3.4.3 Classifying Drug Response of Individual Lines......................... 27

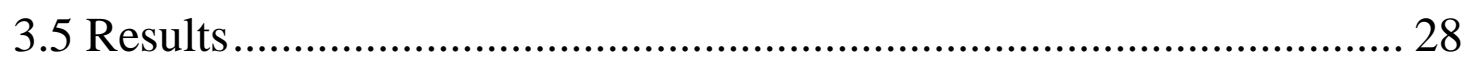

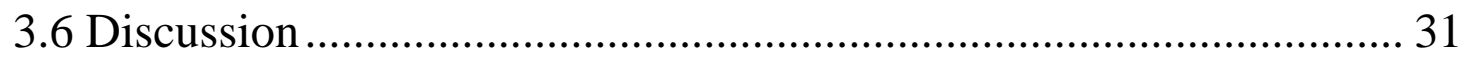

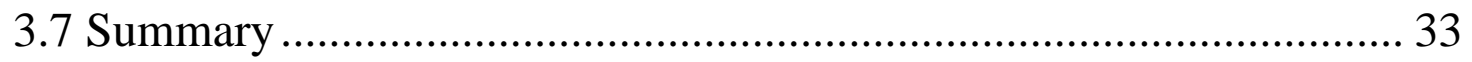

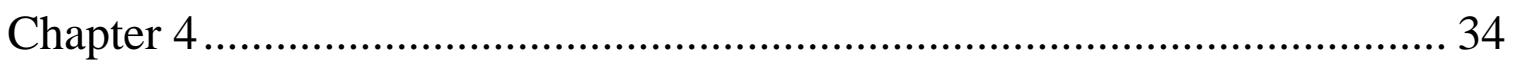

An Integrated Genomic and Proteomic Approach to Chemosensitivity

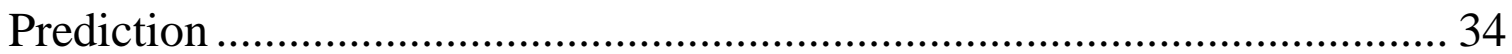

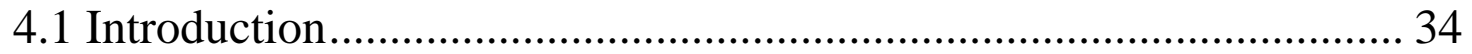

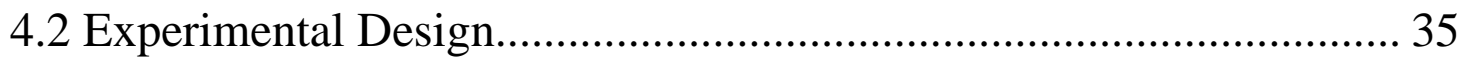

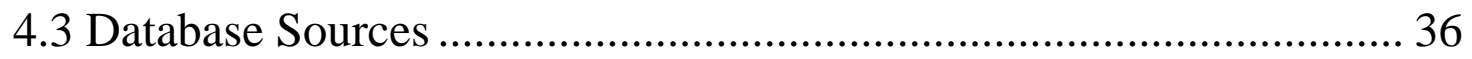

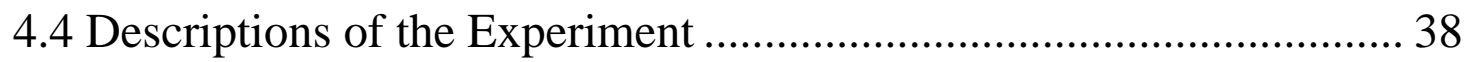

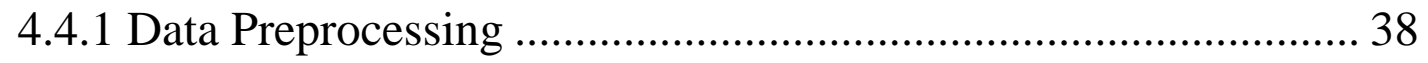

4.4.2 Selecting Gene and Protein Predictors ........................................ 39

4.4.3 Classifying Drug Response of Individual Cell Lines .................. 43

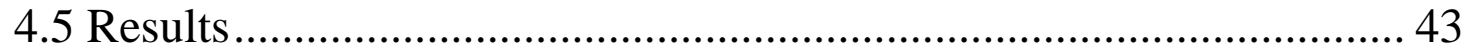

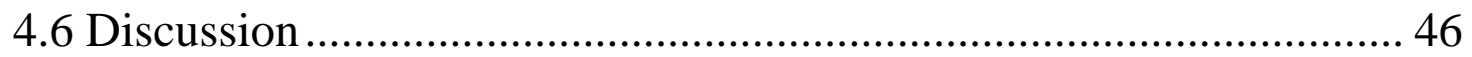

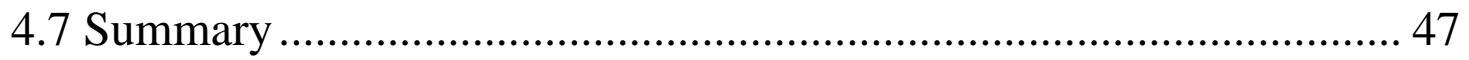

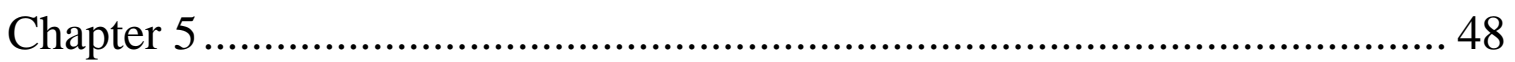

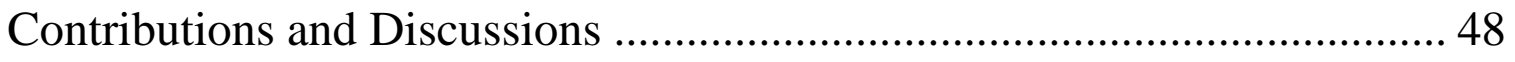

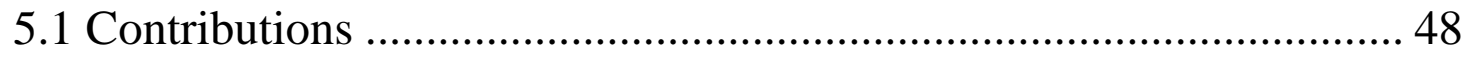

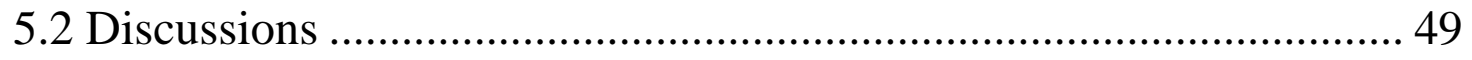

Appendix A: Detailed Results of the Integrated Gene-Protein

Expression-Based Chemosensitivity Classifiers ......................................... 50

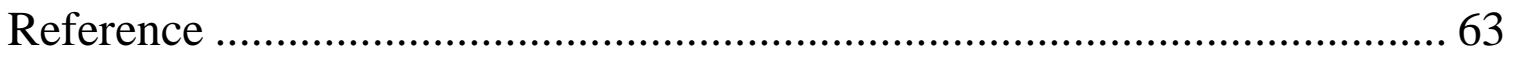




\section{List of Figures}

Figure 3. 1: Model system of predicting cancer drug response by proteomic profiling. 20

Figure 3. 2: Chemosensitivity profiles of different cancer types (using 0.5 SDs as cutoff)

Figure 3. 3: Chemosensitivity profiles of different cancer types (using 0.6 SDs as cutoff)

Figure 3. 4: Chemosensitivity profiles of different cancer types (using 0.8 SDs as cutoff). 24

Figure 3. 5: Comparison of intermediate range drug responses in different cancer types using different cutoff points.................................................. 25

Figure 3. 6: Prediction accuracy of the constructed optimal classifiers ....... 29 Figure 3. 7: Histogram of $P$-values generated from method 1 ..................... 30 Figure 3. 8: Histogram of $P$-values generated from method II .................... 31 Figure 4. 1: Model system of the integrated genomic and proteomic

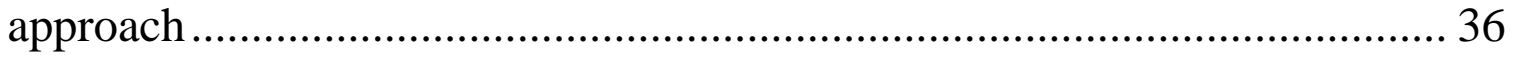

Figure 4. 2: Drug response prediction using proteomic and genomic profiling by method I . 41

Figure 4. 3: Drug response prediction using proteomic and genomic profiling by method II

Figure 4. 4: Prediction accuracy of the constructed optimal classifiers by method I

Figure 4. 5: Prediction accuracy of the constructed optimal classifiers by method II

Figure 4. 6: Final prediction accuracy of the constructed optimal classifiers 
Figure 4. 7: Improvement of the integrated molecular chemosensitivity classifiers over protein expression-based classifiers ................................... 45 


\section{List of Tables}

Table 3. 1: Protein Expression Data Description ........................................ 21

Table 3. 2: Drug Activity Data Description .................................................. 22

Table 3.3: Frequency of $P$-values generated from method 1 ...................... 30

Table 3.4: Frequency of $P$-values generated from method II....................... 31

Table 4. 1: Gene Expression Data Description.............................................. 37

Table A. 1 Number of Selected Features in the Integrated Gene-Protein

Expression-Based Chemosensitivity Classifiers ........................................ 50

Table A. 2 Names of Selected Features in the Integrated Gene-Protein

Expression-Based Chemosensitivity Classifiers ........................................ 56 


\section{Chapter 1}

\section{Introduction}

The ability to accurately predict an individual patient's chemosensitivity will help physicians to choose the most efficient treatment regimens for their cancer patients. Cancer-related genomic and proteomic signatures may provide valuable information about the development and progression of these deadly diseases. Such information will enhance our understanding of fundamental cancer mechanisms and may allow us to accurately predict chemosensitivity

Recent studies on chemosensitivity prediction have focused on transcriptional profiling (1-5). However, transcriptional profiling may not fully reveal the mechanisms of chemosensitivity and does not always correlate with protein profiling (3). Since it is the proteins that ultimately play an essential role in the development and progression of cancer, it is likely that proteomic profiling will yield more direct answers to functional and pharmacologic questions (6). Our first aim is to explore whether proteomic signatures of untreated cancer cell lines could accurately predict their chemosensitivity

Although proteomic and genomic profiles have been generated in cancer profiling, a challenge exists in how to integrate them to predict chemosensitivity. Multi-leveled molecular signatures are important to understand the fundamental cancer mechanisms and to predict biological cellular behaviors. A few studies have been done in which proteomic and genomic profiles have been integrated in cancer profiling (6-8). And these studies used biomarkers with correlated RNA and protein expression patterns (8). The uncorrelated gene and protein expression profiles were removed (8). The artificial 
removal of the uncorrelated molecular markers potentially omits important biological processes in cancer progression and chemosensitivity. Therefore, a novel algorithm is needed to systematically integrate proteomic and genomic profiling without artificially removing uncorrelated biomarkers to predict chemosensitivity.

Furthermore, we sought to develop a novel algorithm to integrate the proteomic and genomic profiles of the cell lines and to classify their chemosensitivity based on the integrated profiles. We also attempted to determine whether the integrated proteomic and genomic profile would increase the chemosensitivity prediction accuracy over the proteomic profile alone. Finally, we sought to explore how several machine learning algorithms performed on our integrated profiling in NCI-60 lines.

This study first developed a machine learning model exclusively based on proteomic profiling to predict drug response. Data on protein expression levels measured by 52 antibodies in a panel of 60 human cancer cell (NCI-60) lines were obtained from previous studies using new high-density reverse-phase protein lysate microarrays (6). Data on the drug responsiveness of these cells lines was obtained from a previous study exploring gene expression and molecular pharmacology (2). We created a new database by merging the proteomic profiles and the drug responses of the NCI-60 lines to the 118 agents. For each drug, the data file contained 52 protein expression variables and 1 drug response. The 52 protein expression variables were predictors, while the drug response was the predicted variable. Random forests in software R, and Relief in software WEKA (9) were used as feature selection methods to select proteomic predictors. Then, the random forests in software $\mathrm{R}$ and nearest neighbor methods in WEKA were used as classification 
methods to classify the chemosensitivity of the cell lines. The prediction results were evaluated by 10 -fold cross validation with WEKA, or by out-of-bag error method with random forests in R. A total of 118 classifiers of the complete range of drug responses (sensitive, intermediate, and resistant) were generated for the evaluated chemotherapy drugs, one for each agent. The accuracy of predicting chemosensitivity to all the evaluated 118 agents was significantly $(P<0.02)$ higher than that of random prediction. The results showed that it was feasible to accurately predict chemosensitivity by proteomic approaches.

Next, we merged genomic profiling into our previous database. For each drug, we formed a new dataset with 1,427 variables, which included 52 protein expression variables and 1,374 mRNA expression variables, as well as 1 drug response. The 52 protein expression variables and 1,374 mRNA expression variables were predictors, while the drug response was the predicted variable. The varSelRF package of random forests in R was used as the feature selection method to select gene and protein predictors. Then, the random forests algorithm in software $\mathrm{R}$ was used as the classification method to classify the cell lines chemosensitivity. The prediction results were evaluated by the out-of-bag error method in bootstrap evaluation (15). The classification accuracy of all the evaluated 118 agents was significantly better $(P<0.001)$ than random prediction accuracy. Furthermore, 76 out of the 118 integrated genomic and proteomic expression-based classifiers could significantly $(P<0.05)$ improve the chemosensitivity prediction accuracy acquired by protein expression-based classifiers alone. Our results showed that the multi-leveled molecular signatures could significantly increase the 
accuracy of drug response prediction.

This thesis is organized as follows. Chapter 2 describes related work. Chapter 3 introduces the machine learning model predicting drug response exclusively based on proteomic profiling. Chapter 4 describes the addition of genomic profiling into our model and how to use the integrated proteomic and genomic profiles to predict drug response. Chapter 5 summarizes our contributions and discusses future work. 


\section{Chapter 2}

\section{Related Work}

\subsection{Introduction}

Chemosensitivity is the susceptibility of tumor cells to the cell-killing effects of anticancer drugs (10). Different tumor cells respond differently to the multitude of chemotherapy agents available (11). If physicians were able to determine which agents were most effective against an individual patient's tumor, therapeutic outcome might ultimately be improved.

How various cancers develop and progress as well as how they respond to various chemotherapy drugs are likely related to their molecular expression patterns. With the development of high-throughput technologies, such as DNA microarray and protein chips, it is possible to efficiently acquire molecular expression data $(12,13)$. Effectively analyzing available molecular expression data and revealing valuable information about cancer development and progression require appropriate machine learning technologies.

The remainder of this chapter is organized as follows. Section 2.2 introduces several machine learning algorithms that were used in this research. Section 2.3 reviews recent research involving molecular expression profiling and machine learning. Section 2.4 discusses some remaining problems in this area and gives brief descriptions of our solutions and contributions. Section 2.5 provides a summary of this chapter.

\subsection{Machine Learning Algorithms}

This section will review the machine learning algorithms that were used in this research and discuss their characteristics in bioinformatics applications. 


\subsubsection{Random forests}

The random forests algorithm (14) is a powerful classification and regression technique that can be used as both a feature selection and a classification algorithm. The random forests algorithm constructs a collection of diverse trees rather than growing a single tree. First, the algorithm selects $n$ bootstraps of samples from the original data. Then, the algorithm builds an ensemble of un-pruned classification trees. Each of these trees is built on a bootstrap of samples. A random subset of all the features is used for splitting the tree nodes. Finally, the classification decision of a test sample is made by majority voting of all the trees. When growing the tree, approximately one-third of the bootstraps of samples are not used. These cases are called out-of-bag cases. These out-of-bag cases can be used as a testing set in model performance assessment. The random forests algorithm can also be used for feature selection. It ranks the importance of features according to their contribution to prediction accuracy (14). If a feature is critical to discriminate samples of different classes, then the classification error will significantly increase when values are randomly rearranged on this feature. We use the error difference between the classification based on the original data and the classification based on randomly permuted data to measure the importance of this feature. In the first part of this study, we ranked protein by the "mean decrease in accuracy" method. Mean decrease in accuracy is performed as follows. For each tree, randomly rearrange the values of the $i^{\text {th }}$ feature for the out-of-bag set, put this permuted set down the tree, and get new classifications for the forest. The error difference between the out-of-bag for the randomly permuted $i^{\text {th }}$ feature and the original out-of-bag error rate is the importance of the $i^{\text {th }}$ feature in "mean decrease 
in accuracy". The larger the error difference, the more important the feature is. The advantage of the random forests algorithm is that the error estimate based on the out-of-bag set is unbiased. Therefore, there is no need to perform cross-validation or use a separate validation set (14). The random forests algorithm has good predictive performance even when most predictive variables are noise, so it is well suited for processing large-scale microarray data and multi-classification problems (15). Furthermore, it can be used when there are more features than samples (15), as is the case in the current study. Another positive feature is that it can be used both for two-class and multi-class problems. This algorithm was used as feature selection method in chapter 3, and classification method in both chapter 3 and 4 .

The varSelRF package recently developed in $\mathrm{R}$ to implement random forests to perform feature selection. The varSelRF package eliminates the least important features in a stepwise fashion, and the out-of-bag error is used to measure the importance of features. First, the algorithm builds a forest with $\mathrm{N}$ trees, and ranks the features according to their importance. Then, it removes the least $20 \%$ important features. Third, it constructs a new forest based on the remaining features and obtains a new out-of-bag error. The algorithm will repeat the previous two steps until two features remain. Finally, the algorithm will choose the feature subset that has the smallest out-of-bag error. The OOB error in each step is used as a feature selection criterion, instead of a model performance measure. The feature subset with the smallest OOB error was selected to build the chemosensitivity classifier. This package was used as feature selection method in chapter 4. 


\subsubsection{Relief}

The Relief algorithm (Kira and Rendell, 1992) is used to estimate the ability of features to distinguish the samples that are near each other. The rationale is that an informative feature should have the same value for samples from the same class and differentiate between samples from different classes. First, the Relief algorithm randomly selects a sample $S$ and finds its two nearest neighbors. One is called nearest hit $\mathrm{H}$, which is from the same class. And the other is nearest miss $\mathrm{M}$, which is from a different class. Next, it updates the value of vector W [F]. W [F] estimates the qualities of each feature in feature subset $\mathrm{F}$ based on the ability of this feature to distinguish the samples that are near each other (16):

$$
W[F]=W[F]-\operatorname{diff}(F, S, H) / m+\operatorname{diff}(F, S, M) / m
$$

Where $W[F]$ is initialized with 0 and $m$ is the number of training samples, which is defined by users. For discrete features the function $\operatorname{diff}(F, s 1, s 2)$ is defined as follows:

$$
\operatorname{diff}\left(F, s_{1}, s_{2}\right)=\left\{\begin{array}{l}
0 ; \operatorname{value}\left(F, s_{1}\right)=\operatorname{value}\left(F, s_{2}\right) \\
1 ; \text { otherwise }
\end{array}\right.
$$

and for continuous features as: (16)

$$
\operatorname{diff}\left(F, s_{1}, s_{2}\right)=\left|\operatorname{value}\left(F, s_{1}\right)-\operatorname{value}\left(F, s_{2}\right)\right| /(\max (F)-\min (F))
$$

The above is the original Relief algorithm. Kononenko (1994) developed an extension, which uses $\mathrm{k}$ nearest misses and hits and their average contribution to W[F], instead of one nearest miss and hit, which was the algorithm used in the current study.

The Relief algorithm can both exploit information locally and provide a global view 
at the same time. The advantage of Relief is that it can process incomplete and noisy data and deal with multi-class problems (16), which makes it suitable to the datasets used in this study.

\subsubsection{IB1}

IB1 is a basic instance-based learner. It is the special case of IBk when $k=1$. IBk implements the k-nearest neighbor algorithm. The k-nearest neighbor algorithm calculates the Euclidean distance or Mahalanobis distance between the test sample and all other labeled samples (17). The test sample is predicted by majority voting from the $\mathrm{k}$ nearest samples. IB1 uses normalized Euclidean distance to find the nearest sample and to predict the test sample into the same class as the nearest sample. If there are multiple samples having the same distance to the test sample, the first one found will be used to predict the test sample (9).

IB1 represents knowledge in the form of specific cases or experiences. The advantage of IB1 is that it is simple to implement and has no parameters to be tuned. It is efficient and successful in many classification problems.

\subsubsection{NNge}

Nearest-neighbor-like algorithm (NNge) is a nearest neighbor method with generalization. It uses non-nested generalized exemplars, which are hyper-rectangular regions of instance space. The hyper-rectangular regions of instance space are used for calculating a 
distance function to classify test instances, which can be viewed as "IF-THEN" rules (9).

It is easy to interpret the knowledge representation from NNge. In addition, NNge is considered to have the least diversions in the datasets per chromatic area (18).

\subsection{Molecular Profiling and Bioinformatics}

The molecular profiles of cancer cells can be determined at the level of mRNA, or protein. DNA microarray is a technology (19) that measures gene expression levels in a biological sample. A collection of DNA spots are attached to a solid surface, such as a silicon chip. These DNA spots serve as probes to indicate whether these particular genes are expressed in the target sample. Traditionally, this technique has been able to examine only a few genes at one time. However, newer technologies now make it possible to measure fifty thousands of genes simultaneously. In addition, it is also possible to analyze many samples in parallel in the same panel. Two of the most widely used platforms are cDNA and oligonucleotide microarrays. Protein microarray is a technique that measures protein expression levels in a biological sample. One type of protein microarray is the reverse-phase protein lysate array. With reverse-phase protein lysate array, samples to be assessed are robotically spotted, and an antibody is then used to measure the amount of a particular protein present in the sample (6).

These molecular expression data have the potential to reveal important information about how cancer develops and progresses as well as how to better detect, diagnose, prevent, and treat the diseases. The challenge lies in how to extract useful cancer-related information from the overwhelming amounts of information. Because molecular 
expression data contain many irrelevant, noisy, and redundant features, it is important to select useful feature subsets to represent all the data according to the goal of the task. A key technology in effectively analyzing molecular expression data involves machine learning. First, machine learning techniques make the classifier that based on the select feature subset, useful in future problems. Second, these techniques can reduce the data size and dimension, which will make the algorithm run faster and more effectively. Third, they can increase the prediction accuracy or make the knowledge representation easy to interpret (20). Machine learning has been used in cancer research for almost 20 years (21). Most often it is used in studies related to cancer detection or diagnosis. It is relatively recently that machine learning has been used in studies exploring chemosensitivity prediction by molecular expression profiling $(12,21-23)$. In the following paragraphs, some related work in this area will be described.

The protein expression data used in this study come from the work of Nishizuka et al's (6). These investigators developed new high-density reverse-phase protein lysate arrays with larger numbers of spots than previously feasible. They applied this method to measure 52 proteins across the NCI- $60^{1}$ cell lines. The NCI-60 lines include human cancer cell lines derived from diverse tissues: brain, blood and bone marrow, breast, colon, kidney, lung, ovary, prostate and skin. They have been used by the National Cancer Institute since 1990 to screen over 100,000 chemical compounds for anticancer activity (6). When the investigators compared the patterns of protein expression with the patterns they had obtained for the same genes at the mRNA level, they found a higher

\footnotetext{
${ }^{1}$ http://www.sanger.ac.uk/genetics/CGP/NCI60/
} 
correlation between cell-structure-related proteins and mRNA than between non-cell-structure related proteins and mRNA. The results indicated that the protein expression measured by their new method highly correlated with transcriptional expression. Therefore, it is reasonable to evaluate the feasibility of using proteomic profiles to predict chemosensitivity.

The Drug Activity Data and gene expression data used in this study was from the work of Scherf et al. (2) which explored whether genomic profiles are related to drug sensitivity. This was the first attempt to integrate large databases on gene expression and molecular pharmacology. In their study, they first employed cluster analyses to group the cell lines based on the gene expression patterns, which were measured in NCI-60 by using cDNA microarrays. Next, they clustered the 60 cell lines using an average-linkage algorithm and a metric based on the growth inhibitory activities $\left(\mathrm{GI}_{50}\right)^{2}$. $\mathrm{GI}_{50}$ is the concentration that inhibits cell growth by $50 \%$ in comparison with untreated controls (2). The $\log _{10}\left(\mathrm{GI}_{50}\right)$ value was used for each compound. They found that the clustering results based on gene expression were different from those based on cell lines’ response to the drugs. They used two clinical agents to exemplify how variations of some gene expressions relate to mechanisms of drug response. They gave the following explanation:” An individual gene can have a major impact on the activities of a large number of drugs but, being just 1 gene out of 1,376, it can have little effect on clustering by gene expression pattern.”

Staunton et al. (3) sought to determine whether the gene expression signatures of

\footnotetext{
${ }^{2}$ http://www.broad.mit.edu/mpr/NCI60/
} 
untreated cells were sufficient to predict chemosensitivity. In their study, they used oligonucleotide microarrays to measure 6,817 gene expression profiles in the NCI-60. They developed their own weighted voting algorithm to classify the cell line chemosensitivity based on gene expression. By using their own genomics-based approach, 88 of 232 expression-based classifiers performed accurately (with $P<0.05$ ) on an independent test set, whereas only 12 of the 232 would be expected to do so by chance. The gene expression-based classifiers were also evaluated by independent datasets. Their results suggested that at least for a subset of compounds genomic approaches to chemosensitivity prediction are feasible.

Varambally et al. (8) sought to characterize and integrate RNA and protein expression data of prostate cancer in order to understand prostate cancer progression with a systems perspective. In their study, they employed an immunoblot approach to derive a prostate cancer proteome. Then, they used unsupervised hierarchical clustering to reveal over 100 proteomic alterations in prostate cancer progression. Third, they developed an integrated model to incorporate qualitative proteomic alterations as assessed by immunoblotting with transcription data. Their analyses indicated that the proteins that were qualitatively concordant with gene expression could be used to define a multiplex gene predictor of clinical outcome. The feasibility of integrating uncorrelated transcriptional profiles and the proteomic profiles to predict chemosensitivity remains to be determined.

Shankavaram et al. (24) sought to evaluate the utility of mRNA profiling to predict protein expression levels. In their study, they used 162 monoclonal antibodies to measure 
the expression of 94 proteins in NCI 60 cell lines by reverse-phase protein lysate array. They generated mRNA profiles using four different microarray platforms (cDNA arrays and Affymetrix HU6800, HG-U95A, and HG-U133A arrays) and then compared and combined these profiles to build a consensus mRNA expression data set with minimal noise and error. Next, they compared the global concordance of expression among the consensus mRNA, individual-platform mRNA, and reverse-phase protein lysate array protein data sets, and employed PAM (nearest shrunken centroid algorithm) in R to assess the performance of protein and RNA data for prediction of tissue function. Lastly, they employed the clustered image map based on categories to assess the protein-transcript correlations for structural proteins. Their results showed that (I) the consensus mRNA set had the highest concordance with protein data; (II) when evaluating the ability of profiles from various platforms to cluster cell lines by tissue of origin, the overall misclassification error rate was lowest for the protein data, followed by the consensus, HG-U133A, HG-U95A, cDNA and (III) analysis based on the Gene Ontology categories showed protein-transcript correlations are particularly high for structural proteins. They concluded that transcript-based technologies continue to be useful, even when the ultimate target is protein, because transcript-based technologies are more mature and can assess larger numbers of genes at one time.

\subsection{Open Problems}

Recent studies investigating methods of predicting chemosensitivity have focused on transcriptional profiling (1-5). However, transcriptional profiling only reveals 
information about mRNA. It is the protein products that play an essential role in cancer development and are the target of the majority of current diagnostic markers and therapeutic agents (6). Therefore, proteomic profiling may yield more direct answers to functional and pharmacologic questions (6). Therefore, the first part of this thesis project was to explore whether a proteomic approach can accurately predict chemosensitivity. In particular, we developed a machine learning model exclusively based on proteomic profiling to predict drug response. The challenge of protein expression-based chemosensitivity prediction is the small amount of available protein expression data and the small size of the samples. Only a few proteomic datasets have been measured on the NCI-60 panel. The protein expression data file we used was generated by Nishizuka et al. (6). However, as a result of difficulties encountered in proteomic technologies, the number of proteins (25) evaluated is relatively small. Furthermore, there are only 60 cell lines in the NCI-60 panel. Therefore, the limited data made it difficult to construct protein expression-based classifiers to predict chemosensitivity. To rank the importance of proteins and select predictors, we used the random forests (§3.3.1) or Relief (§3.3.2) algorithms. Both algorithms have good performance on the noisy and incomplete data. To evaluate the prediction performance, we used either a bootstrapped out-of-bag error (14) or a 10-fold cross-validation method. Both methods provide an unbiased evaluation of prediction performance $(14,26)$.

Another challenge in this research area is how to integrate proteomic profiling with genomic profiling to predict chemosensitivity. Previous studies (6-8) that have attempted to integrate genomic and proteomic information, have focused on the correlation between 
gene and protein expression patterns. Only the markers whose RNA and protein expression patterns correlated with each other were included in the prediction model; The uncorrelated gene and protein expression profiles were removed (8). The artificial removal of the discordant molecular markers potentially omitted some important biological information regarding chemosensitivity mechanisms, such as protein-protein interactions and protein-gene interactions. To address this limitation, we developed a novel algorithm to integrate both protein expression and gene expression profiles. Such an algorithm can be used to systematically evaluate genome-wide DNA, RNA, and protein contributions in chemotherapy sensitivity. The challenge of constructing integrated gene-protein expression-based chemosensitivity classifiers is the unbalanced data. The proteomic expression profiles we used in this study contain 52 proteomic features (§3.3), while the genomic expression profiles we used contain 1,374 genomic features (§4.3). Therefore, there are considerably fewer proteomic features than genomic features. To account for the unbalanced gene expression and protein expression profiles, we developed two stepwise feature selection schemes to identify the integrated gene and protein signatures. We then used a random forests algorithm to construct chemosensitivity classifiers based on these signatures.

\subsection{Summary}

This chapter reviewed recent studies that used machine learning technologies and molecular expression data in the area of cancer research. Then, several machine learning algorithms that were used in the current study were described. Finally, current challenges in using molecular profiling and machine learning technologies in the prediction of 
chemosensitivity were discussed. Our approaches to overcome those challenges were briefly described. A more detailed description of this work will be provided in the subsequent chapters. 


\section{Chapter 3}

\section{Predicting Cancer Drug Response by Proteomic Profiling}

\subsection{Introduction}

Accurately predicting an individual patient's response to anticancer drugs would be very helpful in the selection of optimal treatments. Recent studies in this area have focused on transcriptional profiling (1-5), but the expression of transcriptional profiling does not necessarily correlate with that of proteomic profiling. Proteins rather than mRNA are the target of the majority of current diagnostic markers and therapeutic agents (6). Therefore proteomic profiling may yield more direct answers to functional and pharmacologic questions (6).

In this chapter, we introduce a machine learning model for predicting drug response exclusively based on proteomic profiling. We sought to determine whether proteomic signatures of untreated cancer cells could accurately predict their chemosensitivity. The accuracy of predicting chemosensitivity to all the evaluated 118 chemotherapeutic agents was significantly higher $(P<0.02)$ than random prediction accuracy. The results showed it was feasible to predict drug response of cancer cell lines by using proteomic profiles.

The remainder of this chapter is organized as follows. Section 3.2 introduces the design of the experiment. Section 3.3 introduces the data sets used in the experiments. Section 3.4 outlines the major steps of the experiments. Section 3.5 provides results and assesses the results. Section 3.6 discusses protein expression-based classifiers. Section 3.7 provides a summary of this chapter. 


\subsection{Experimental Design}

We set out to determine whether we could use the protein profiles of a panel of 60 human cancer cell (NCI-60) lines to create a model that would accurately predict their responsiveness to 118 anticancer drugs. The experiment was designed as follows: First, to construct the supervised protein expression-based classifiers for the prediction of drug response, we created a new database by merging the proteomic profiles and the responses of the NCI-60 lines to the 118 agents. For each drug, the data file contained 52 protein expression variables and 1 drug response. The 52 protein expression variables were predictors, while the drug response was the predicted variable. We partitioned the numeric drug response into three classes: sensitive, intermediate, and resistant. Second, we used a random forests algorithm in software $\mathrm{R}$, and a Relief algorithm in software WEKA as feature selection methods to select proteomic predictors. Third, random forests in software $\mathrm{R}$ and nearest neighbor methods in WEKA were used as classification methods to classify the cell lines’ chemosensitivity according to the selected predictors. The prediction results using the WEKA techniques were evaluated by 10 -fold cross validation, and those using random forests were evaluated by the out-of-bag error method with R. Finally, $P$-values were calculated to evaluate the statistical significance of the prediction results. The experiment is outlined in Figure 3.1. 


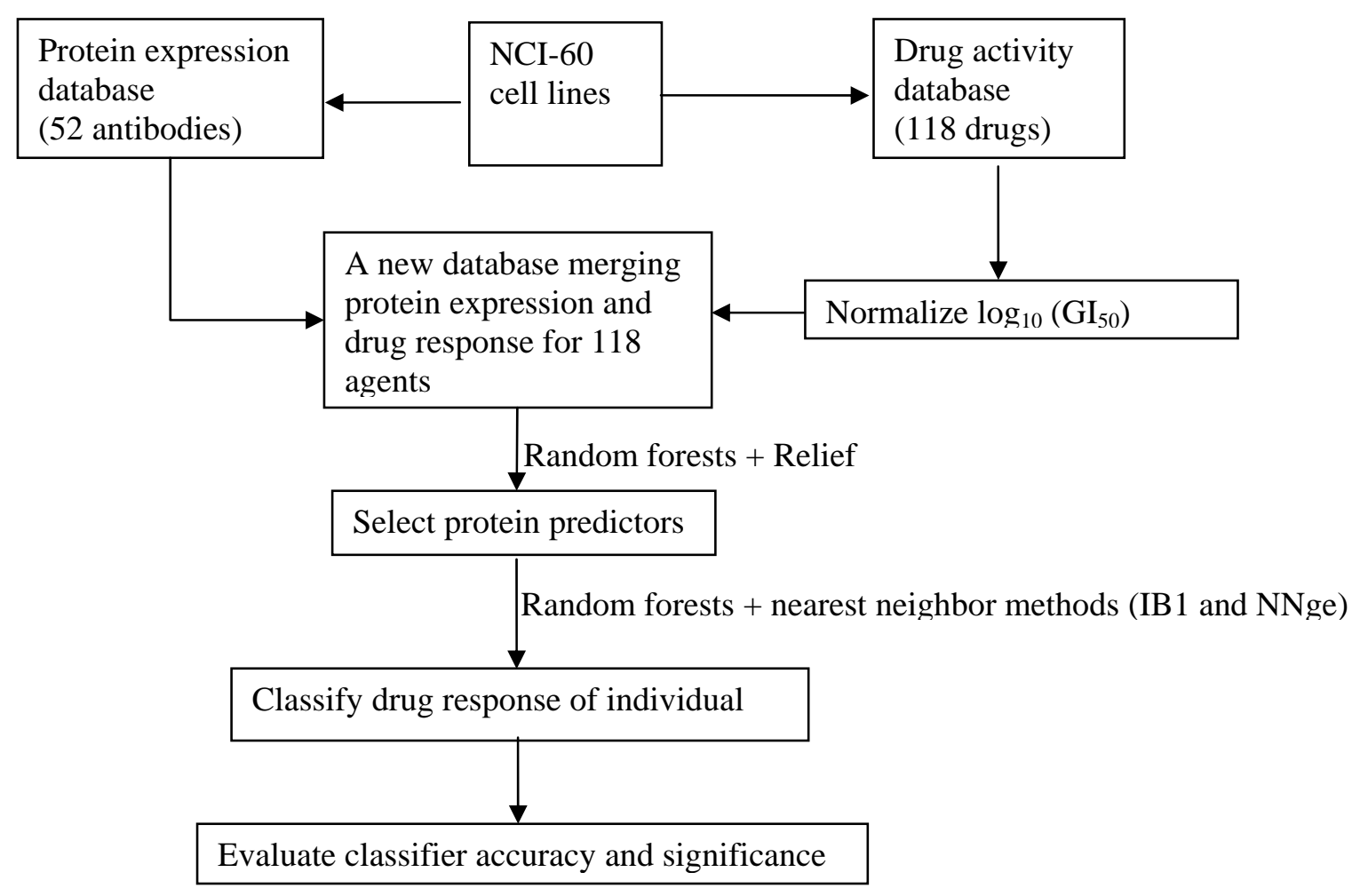

Figure 3. 1: Model system of predicting cancer drug response by proteomic profiling

\subsection{Database Sources}

In this study, we used two data files from the NCI DISCOVER database ${ }^{3}$. Following are descriptions of the datasets.

\section{Protein Expression Data}

Nishizuka et al. generated the protein expression data file (6). Their study was discussed in §2.3. The investigators developed a protocol for reverse-phase protein lysate arrays that can measure a larger number of spots than previously feasible. They employed P-SCAN and a quantitative dose interpolation method to measure the expression of 52

\footnotetext{
${ }^{3}$ http://discover.nci.nih.gov/
} 
proteins across the NCI-60 human cancer cell lines (§2.3). The data file is available online. ${ }^{4}$ The protein expression data is described in Table 3.1.

Table 3. 1: Protein Expression Data Description

\begin{tabular}{|l|l|}
\hline Column A: & HUGO (Human Genome Organisation) name \\
\hline Column B: & Antibody name \\
\hline Column C: & Vendor of antibody \\
\hline Columns D-BK: & Protein expression values on the 60 human cancer cell lines \\
\hline
\end{tabular}

\section{Drug Activity Data}

Scherf et al. generated the drug activity profiles of the NCI-60 lines against 118 anti-cancer agents (2). They used a sulphorhodamine B assay to evaluate changes in total cellular protein after 48 hours of drug treatment as a measure of growth inhibition. Drug activities ( $\left.\log _{10} \mathrm{GI}_{50}\right)$ were recorded for each of the 60 human cancer cell lines. $\mathrm{GI}_{50}$ (§2.3) is the concentration that inhibits cell growth by $50 \%$ when compared with untreated controls. For each agent, the drug activity profile consists of 60 drug activity values, one for each cell line. The drug activity profiles of the 118 agents are available online ${ }^{5}$. Table 3.2 briefly described the data.

\footnotetext{
${ }^{4}$ http://discover.nci.nih.gov/host/2003_profilingtable7.xls

5 http://discover.nci.nih.gov/nature2000/data/selected_data/dataviewer.jsp?baseFileName=a_matrix118\&ns $\mathrm{c}=2 \&$ dataStart $=3$
} 
Table 3. 2: Drug Activity Data Description

\begin{tabular}{|l|l|}
\hline Column A: & Mechanism of action \\
\hline Column B: & Drug name \\
\hline Column C: & NSC number \\
\hline $\begin{array}{l}\text { Column } \\
\text { D-BK: }\end{array}$ & Drug activities (- $\log _{10} \mathrm{GI}_{50}$ ) across 60 human cancer cell lines. \\
\hline
\end{tabular}

\subsection{Descriptions of the Experiment}

\subsubsection{Defining Cutoffs of Drug Sensitivity and Resistance}

We investigated the drug activity profiles of these 118 anti-cancer agents using different cutoffs to define drug responses. In the literature, there is no formal definition of how many standard deviations (SDs) away from the mean should be used as the cutoff to define drug response.

In our study, the values of $\log _{10}\left(\mathrm{GI}_{50}\right)$ were normalized across the NCI-60 lines. We then tried 3 different cutoffs to define drug responses of the NCI-60 lines: 0.5 SDs, 0.6 SDs, and 0.8 SDS. For example, for each drug, when we used 0.5 SDs as the cutoff, cell lines with $\log _{10}\left(\mathrm{GI}_{50}\right)$ 0.5 SDs above the mean were defined as resistant to that drug; those with $\log _{10}\left(\mathrm{GI}_{50}\right)$ 0.5 SDs below the mean were defined as sensitive; and the remaining cell lines were defined as intermediate. In NCI-60 lines, there are several cancer types. Therefore, we averaged the profiles on the cell lines for each cancer type, and summarized the chemosensitivity profiles by cancer type. Results are shown in Figure 3.2 (0.5SDs as cutoff), Figure 3.3 (0.6 SDs as cutoff), and Figure 3.4 (0.8 SDs as cutoff). 


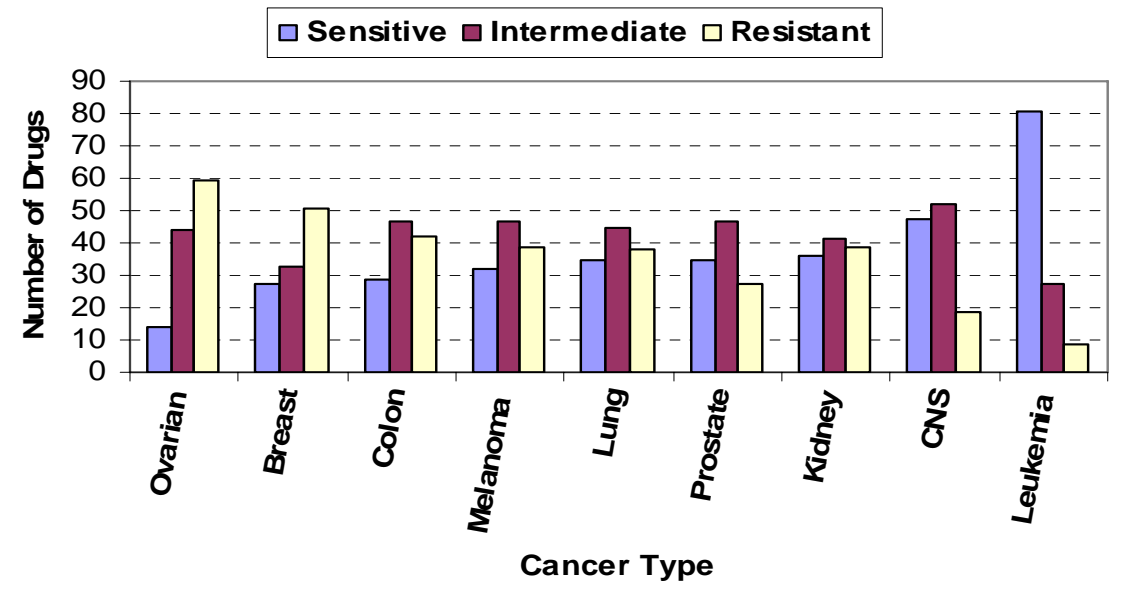

Figure 3. 2: Chemosensitivity profiles of different cancer types (using 0.5 SDs as cutoff)

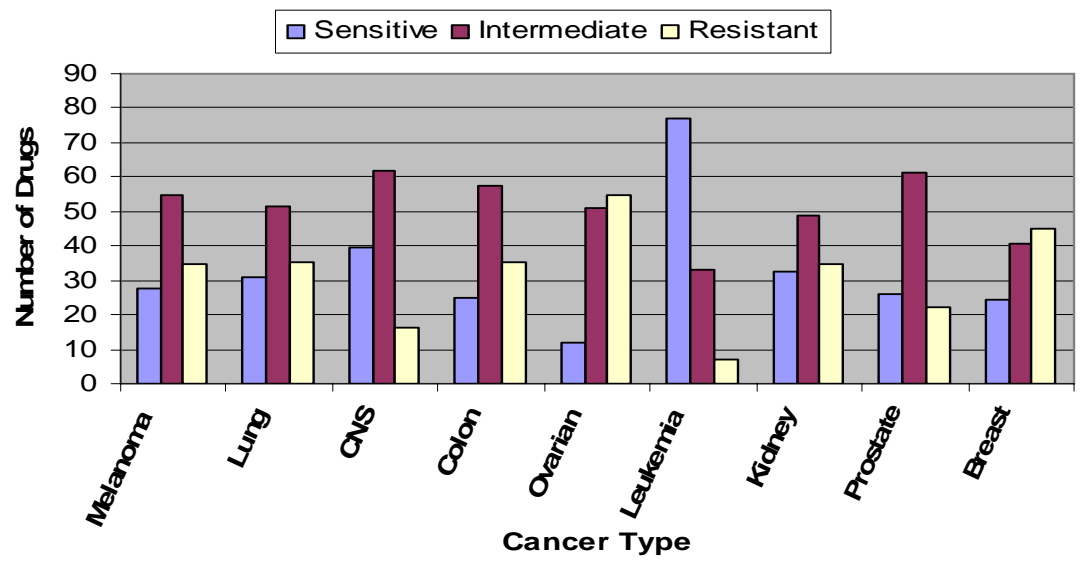

Figure 3. 3: Chemosensitivity profiles of different cancer types (using 0.6 SDs as cutoff)

A cutoff of 0.8 SDs was used by Staunton et al. (3) in their study to define drug response, possibly because many of the chemical compounds they screened have not yet been approved as anti-cancer drugs and may exhibit a wide range of chemical activities. 
However, all 118 drugs used by Scherf et al. to generate the profiles we used in our study are either approved or potential anti-cancer drugs. They have a narrower range of drug activities in the NCI-60 lines. Therefore, when we tried 0.6 SDs and 0.8 SDs as the cutoff, the drug response distribution was unbalanced. The majority of drugs fell into the intermediate level (Figure 3.4). The machine learning algorithms tend to minimize overall prediction error by sacrificing the minor classes' prediction accuracy. When we used 0.6 SDs and 0.8 SDs as cutoffs, sensitive and resistant levels were minor classes. The algorithm predicted some instances of sensitive and resistant levels as intermediate levels in order to favor the overall prediction accuracy. Such a model may cause serious problems in clinical application. For example, some patients, who actually are sensitive to a drug, may be predicted to have an intermediate response. As a result, they may not be prescribed the most effective chemotherapeutic regimen.

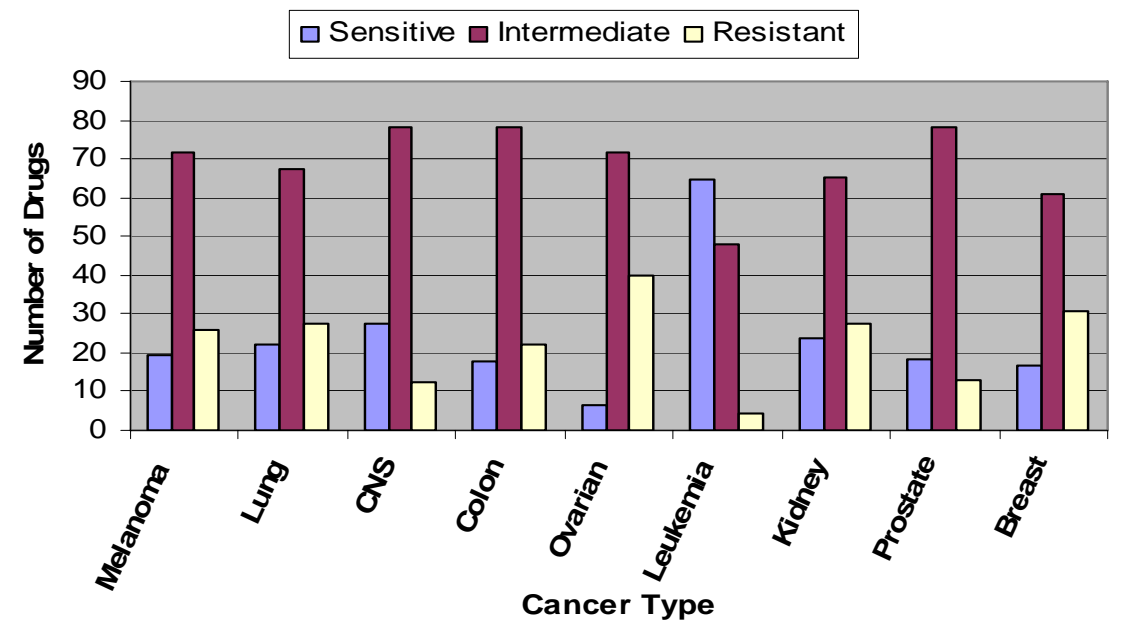

Figure 3. 4: Chemosensitivity profiles of different cancer types (using 0.8 SDs as cutoff) 
We calculated and compared the percentage of intermediate level responses for each cancer type using 0.5 SDs, 0.6 SDs and 0.8 SDs as the cutoff, as shown in Figure 3.5. Using 0.6 SDs or 0.8 SDs caused an unbalanced distribution of drug responses. However, when we used 0.5 SDs as the cutoff, the percentage of intermediate level responses was about one third, giving the most balanced distribution of drug responses. In order to build a chemosensitivity prediction model feasible for future clinical application, we chose 0.5 SDs as the cutoff for defining drug response.

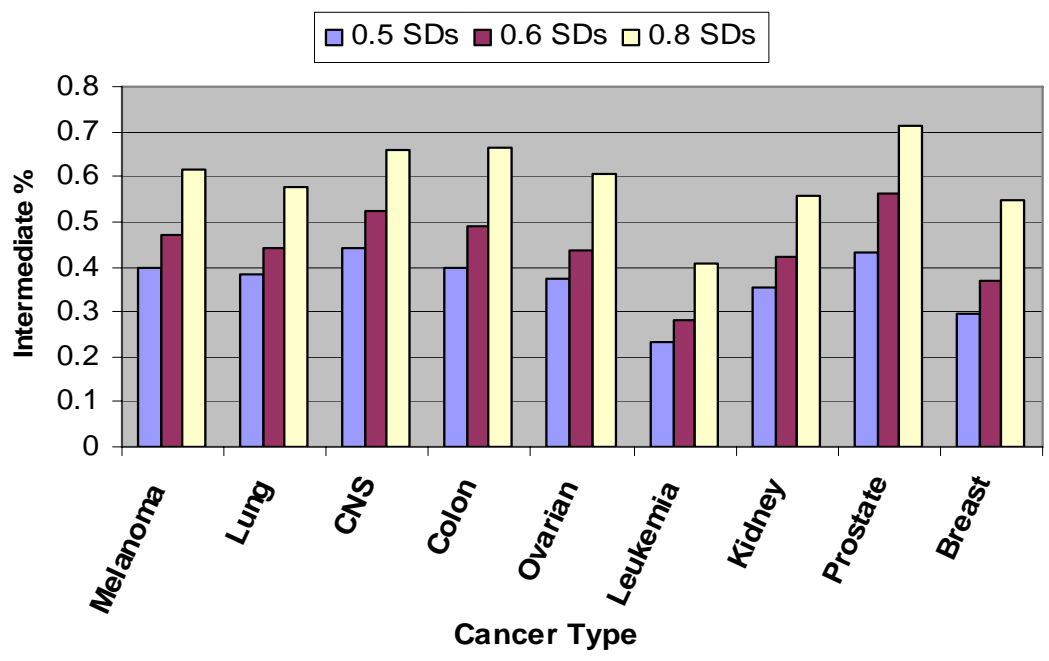

Figure 3. 5: Comparison of intermediate range drug responses in different cancer types using different cutoff points

Furthermore, in the study by Staunton et al. (3), they used only binary classifiers: sensitive and resistant. We improved upon those methods by incorporating an intermediate level into the drug responses. The multi-class definition of drug response may be more appropriate and help us to better understand the mechanisms of 
chemosensitivity.

\subsubsection{Selecting Protein Predictor}

As we discussed in §2.3, appropriate feature selection is a prerequisite of building good classifiers. Here, we ranked the proteins by using the "mean decrease in accuracy" method of random forests (§2.2.1) and selected the top ranking proteins as the predictors. First, the random forests algorithm was run on all 52 proteins and an output of importance ranking of proteins for each drug was generated. Next, the lowest ranking proteins were filtered in a stepwise way. The bottom 2 proteins were first removed and the remaining 50 proteins were used for the prediction. Then, the bottom 5 proteins were removed each successive time. When there were 10 proteins left, the bottom 1 protein was removed each successive time. Finally, we got 16 different subsets of variables for each drug. In this study, the smallest subset consisted of 3 proteins.

When the random forests algorithm failed to achieve an accuracy $>50 \%$ in drug response prediction, we used the Relief (§2.2.2) method in WEKA 3.4 to rank the proteins and selected the top ranking proteins as the predictors. For each drug, the Relief algorithm obtained an importance ranking of all 52 proteins. Next, the lowest ranking proteins were filtered in a stepwise manner. The bottom one protein was removed each time. The process was repeated until only one protein was left. 


\subsubsection{Classifying Drug Response of Individual Lines}

For those drug datasets whose protein predictors were ranked by the random forests, we ran the random forests algorithm, which was described in \$3.4.2, as the classification method on every subset of variables. We got 16 different subsets of proteins, so the random forests algorithm was run 16 times, one for each subset. The prediction accuracy along with the protein list was recorded. We chose the one with the smallest number of proteins and the highest prediction accuracy as the optimal classifier.

The prediction accuracy by the random forests algorithm was evaluated by the bootstrapped out-of-bag error method. The bootstrapped out-of-bag method uses two thirds of the samples as the training set and the remaining samples as the validation set. Since this method has been proven to be unbiased (14), we did not need to perform cross-validation or use an independent dataset to evaluate the results.

For those drug datasets whose protein predictors were ranked by the Relief method, several WEKA classifiers were explored to classify the cell lines. For each drug in the experiment, a new subset of proteins was obtained after removing the bottom protein one at a time. A WEKA classifier was run on the new feature subset to get the prediction accuracy. The process was repeated until only one protein was left. We then identified the optimal classifier with the highest prediction accuracy along with the protein predictors for each drug. Nearest neighbor methods (IB1§2.2.3 and NNge §2.2.4) in WEKA performed best in this study. The chemosensitivity prediction accuracy for the following four drug were significantly improved: Clomesone (NSC: 338947), Camptothecin, 10-OH (NSC: 107124), Camptothecin, 20-ester (S) (NSC: 606985), and Floxuridine 
(FUdR) (NSC: 27640).

The prediction accuracy by the WEKA classifiers was evaluated by using 10 -fold cross validation. In 10 -fold cross validation, the data set is first randomly partitioned into 10 folds. The first 9 folds are of equal size and the last fold contains the remaining samples. The prediction model uses the 9 folds as training data and the remaining one as testing data. The process is repeated 10 times so that each fold is used as the testing data one time. The overall prediction accuracy is the average prediction accuracy of all 10 testing data. We used 10-fold cross validation to evaluate the prediction models in this study because the estimation accuracy by this validation method has been proven to have the lowest bias and variance among all validation methods (26). As a result, it provides an objective evaluation of the performance of our prediction models in general.

\subsection{Results}

Overall, the optimal classifiers used between 3 and 26 protein predictors. The average number of protein predictors for all 118 classifiers was 8 . The overall prediction accuracy of the optimal classifiers for the 118 drugs is summarized in Figure 3.6. Detailed results were available from Clinical Cancer Research supplementary web site. (27) 


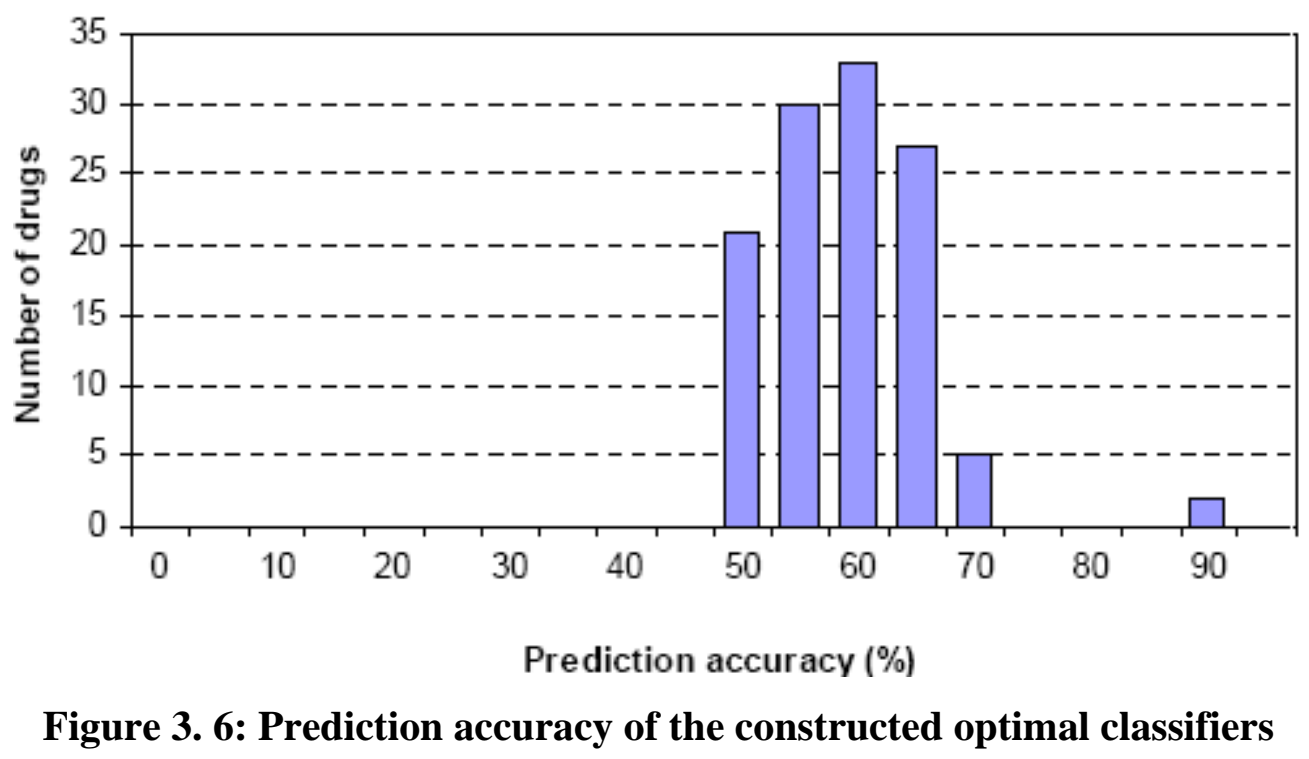

To assess the significance of our prediction results based on the protein profile (Figure 3.6), we used two methods to demonstrate that our prediction results were significantly better than random prediction. In the first method, we kept the original class labels' distributions and randomly permuted them for each drug. For instance, a drug is examined on 60 cell lines. There are 17 resistant cell lines, 22 intermediate cell lines, and 21 sensitive cell lines. Then, the 60 class labels were randomly rearranged without changing the class distribution (17 resistant, 22 intermediate, and 21 sensitive). We calculated the random prediction accuracy by using the percentage of matches between the original class labels and permuted class labels. This calculation was repeated 1000 times. The upper percentile of our prediction accuracy in the 1000 random prediction results was used to calculate $P$-values. If the prediction accuracy of our optional classifier was better than the $95^{\text {th }}$ percentile of those 1000 random prediction accuracies, we concluded that our prediction was significantly better than random prediction $(P<0.05)$. The results of method 1 are shown in Table 3.3 and Figure 3.7. 
Table 3.3: Frequency of $\boldsymbol{P}$-values generated from method 1

\begin{tabular}{|l|l|l|l|l|l|l|l|l|l|}
\hline $\boldsymbol{P}$-value & 0 & 0.001 & 0.002 & 0.003 & 0.004 & 0.005 & 0.006 & 0.007 & 0.019 \\
\hline Frequency & 97 & 9 & 4 & 1 & 1 & 3 & 1 & 1 & 1 \\
\hline
\end{tabular}

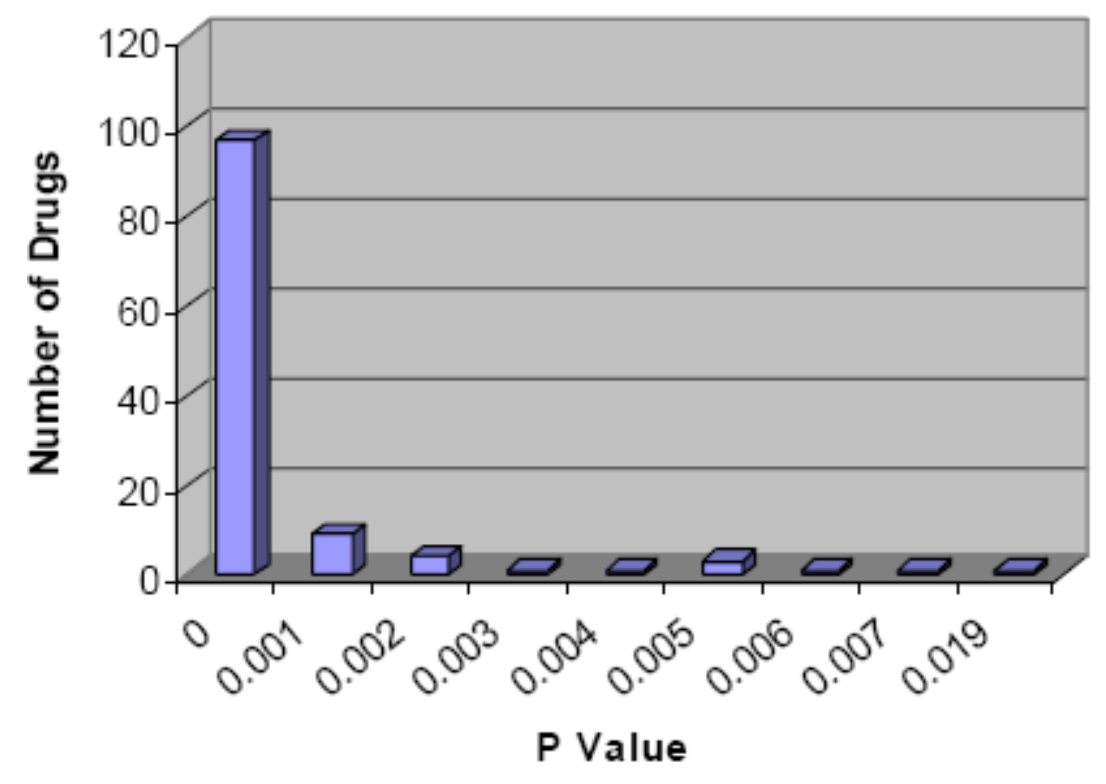

Figure 3. 7: Histogram of $P$-values generated from method 1

In the second method, the class labels distribution for each drug was not fixed, as it was in the first method. For each drug, we randomly assigned a class label (sensitive, resistant, intermediate) to each cell line. Then, we calculated the random prediction accuracy by using the percentage of matches between the original class labels and randomly assigned class labels. This calculation was repeated 1000. The upper percentile of our prediction accuracy in the 1000 random prediction results was used to calculate $P$-values. If the prediction accuracy of our optional classifier was better than the $95^{\text {th }}$ percentile of those 1000 random prediction accuracies, we concluded that our prediction 
was significantly better than random prediction $(P<0.05)$. The results of method 2 are shown in Table 3.4 and Figure 3.8.

Although $P$-values by the second method were more significant than that by the first method, the second method did not take the unbalanced class labels distribution into account. Therefore, we used the first method to assess our prediction results.

Table 3.4: Frequency of $\boldsymbol{P}$-values generated from method II

\begin{tabular}{|l|l|l|l|l|l|l|}
\hline $\boldsymbol{P}$-value & 0 & 0.001 & 0.002 & 0.005 & 0.006 & 0.008 \\
\hline Frequency & 105 & 7 & 2 & 1 & 2 & 1 \\
\hline
\end{tabular}

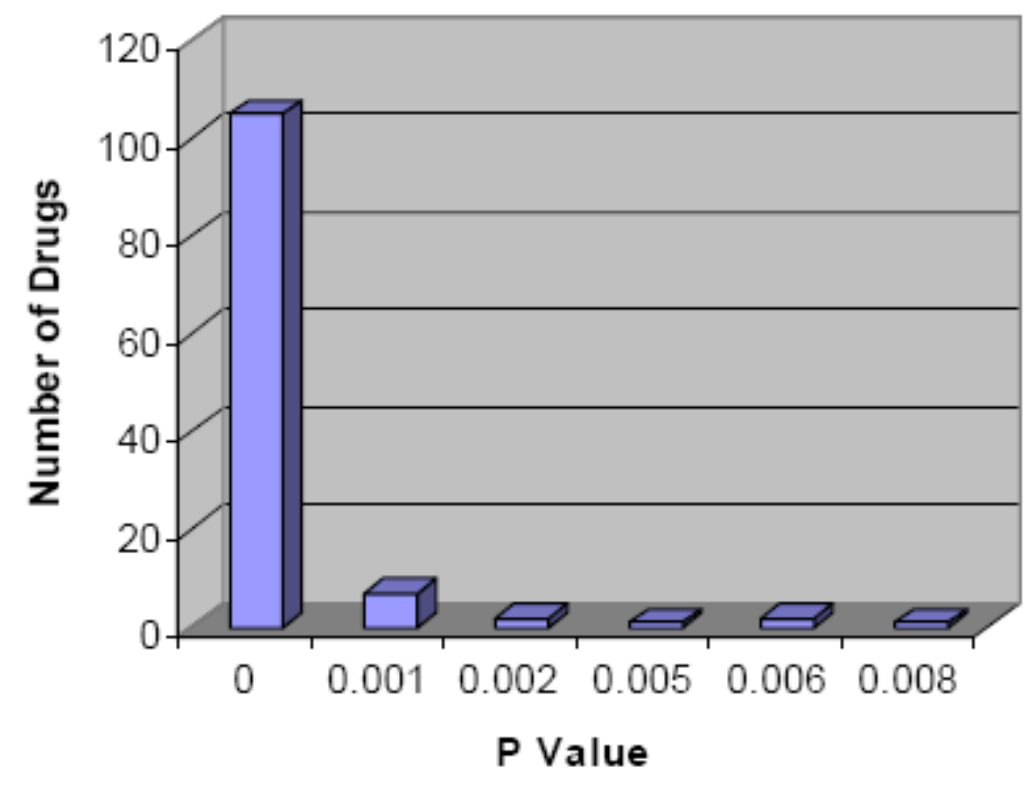

Figure 3. 8: Histogram of $\boldsymbol{P}$-values generated from method II

\subsection{Discussion}

A particular challenge in a protein expression-based chemosensitivity study is the small amount of available protein expression data, the result of difficulties associated with 
proteomics technology (6). We used the dataset from Nishizuka et al. (6). The small number of proteins (25) and the limited number of cell lines made it difficult to construct protein expression-based classifiers to predict chemosensitivity. To rank the importance of proteins and select predictors, we used a random forests (§3.3.1) or Relief (§3.3.2) algorithm. Both algorithms performed well on the noisy and incomplete data. To evaluate the prediction performance, we used either a bootstrapped out-of-bag error method (20) or 10-fold cross-validation method. A disadvantage of both evaluation methods is that they further reduce the size of the samples used to construct the classifiers. Therefore, the prediction accuracy evaluated by these two methods could be potentially compromised. However, both methods provide an unbiased evaluation for the prediction of performance (14, 26). In addition, we constructed multi-class (sensitive, resistant, and intermediate) chemosensitivity classifiers rather than binary ones in this study. Although a multi-classification algorithm is more difficult than a binary one and tends to compromise prediction accuracy (28), it includes the complete range of drug responses and may reveal important mechanistic information.

Considering these challenges and limitations, the prediction accuracies we achieved are notable. The chemosensitivity prediction accuracy of all the 118 evaluated agents is statistically significantly $(P<0.02)$ better than random prediction accuracy. Specifically, 117 agents reached the significance level at $P<0.007$ and the remaining one at $P<0.019$. Our results showed that it was feasible to construct accurate chemosensitivity classifiers using a dataset of 60 instances by 52 protein expression features. 


\subsection{Summary}

In this chapter, we described how we developed a machine learning model system to classify cell line chemosensitivity exclusively based on proteomic profiling. We used a dataset containing protein expression levels of 52 proteins in a panel of 60 human cancer cell (NCI-60) lines obtained by new high-density reverse-phase protein lysate array technology. We employed random forests and nearest neighbor methods as classifiers, and the bootstrapped out-of-bag error or 10-fold cross method to get unbiased validation. We also employed a dataset detailing the response of the NCI-60 lines to 118 anticancer drugs. The accuracy of chemosensitivity prediction of all the evaluated 118 agents was significantly higher $(P<0.02)$ than that of random prediction. Our results showed that it was feasible to predict drug response of cancer cell lines by proteomic profiling. 


\section{Chapter 4}

\section{An Integrated Genomic and Proteomic Approach to Chemosensitivity Prediction}

\subsection{Introduction}

Pharmacologically interesting behaviors are not always reflected at the transcriptional level. It may be necessary to analyze cellular functions at different levels. Integrating genomic and proteomic profiles is an important approach to understanding fundamental cancer mechanisms and to predicting biological cellular behaviors. Such an approach may be necessary to accurately reveal the biological nature of cancer progression and chemosensitivity.

In the previous chapter, we described how we built a machine learning model exclusively based on proteomic profiling and demonstrated that it was feasible to predict the drug responsiveness of cancer cell lines by a proteomic approach. In this chapter, we describe how we extended the model by integrating genomic profiling with proteomic profiling. We developed a novel feature selection algorithm to identify the genomic and proteomic signatures and constructed chemosensitivity classifiers based on the integrated profiles. We sought to determine whether the integrated profiles were able to further enhance the performance of chemosensitivity prediction acquired by protein expression-based classifiers. The classification accuracy of all the evaluated 118 chemotherapeutic agents was significantly better $(P<0.001)$ than random prediction. Seventy-six out of the 118 classifiers significantly $(P<0.05)$ improved the chemosensitivity prediction accuracy acquired by protein expression-based classifiers 
alone. These results demonstrate that our integrated genomic and proteomic approach could further increase chemosensitivity prediction accuracy.

The remainder of this chapter is organized as follows. Section 4.2 introduces the design of the experiment. Section 4.3 introduces the data sets used in the experiments. Section 4.4 outlines the major steps of the experiments. Section 4.5 presents the results. Section 4.6 discusses integrated profiling-based classifiers. Section 4.7 provides a summary of this chapter.

\subsection{Experimental Design}

We predicted drug response of a panel of 60 human cancer cell (NCI-60) lines to 118 anticancer drugs. The experiment was designed as follows: First, for each drug, we formed a new dataset with 1,427 variables after data preprocessing and data quality control, which included 52 protein expression variables, 1,374 mRNA expression variables, and 1 drug response. The 52 protein expression variables and 1,374 mRNA expression variables were predictors, while the drug response was the predicted variable. Second, the varSelRF package of random forests in $\mathrm{R}$ was used as a feature selection method to select gene and protein predictors. Third, random forests algorithms in software R were used to classify the cell lines' chemosensitivity according to the selected predictors. The prediction results were evaluated by the out-of-bag error method in bootstrap evaluation (15). Finally, $P$-values were calculated to evaluate the significance of the prediction results. The experimental design is outlined in Figure 4.1. 


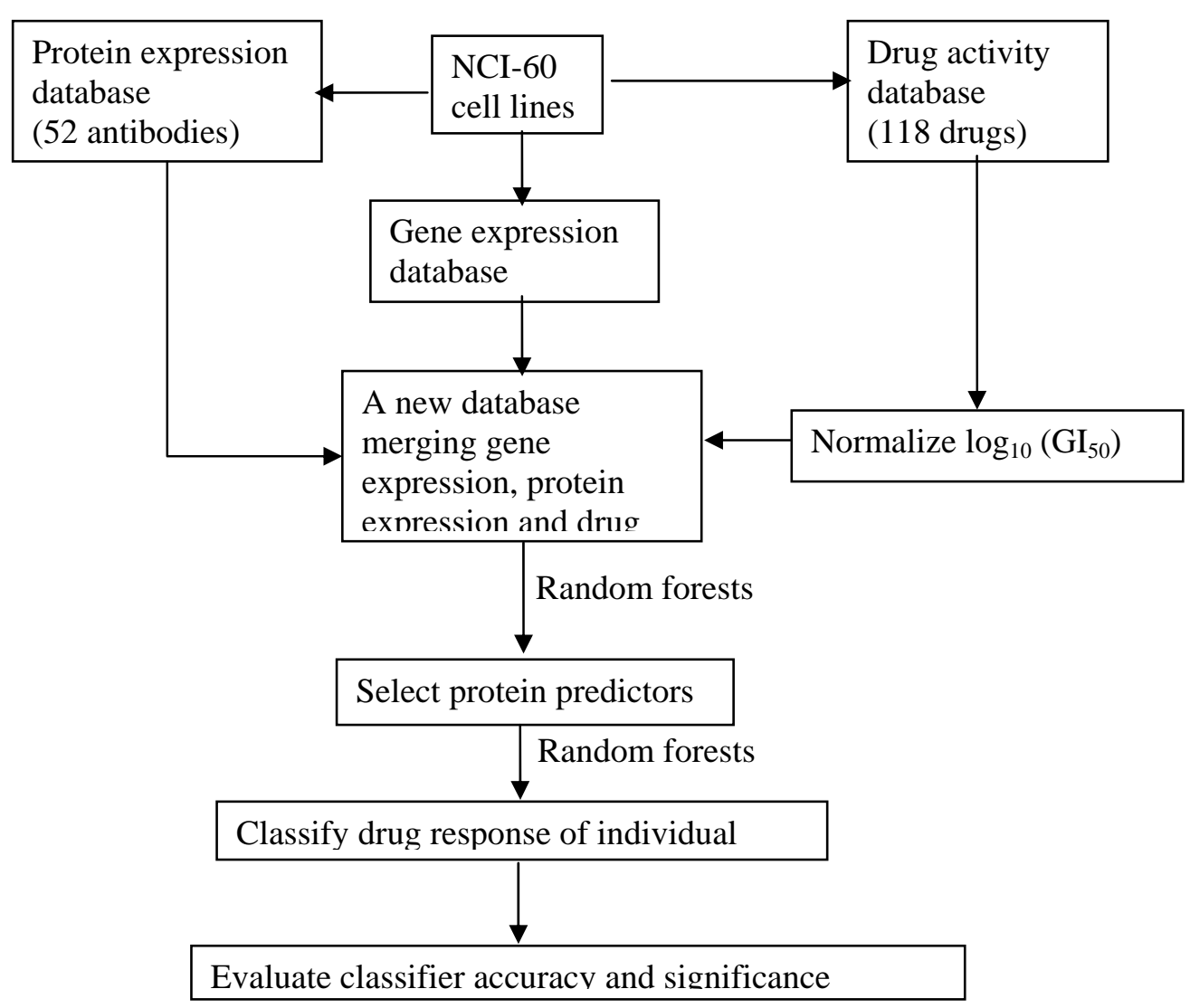

Figure 4. 1: Model system of the integrated genomic and proteomic approach

\subsection{Database Sources}

In this study, we used three data files from the NCI DISCOVER database ${ }^{6}$. Two of them, Protein Expression Data and Drug Activity Data were described in §3.3. The third one is the gene expression data, which is described below.

\footnotetext{
${ }^{6}$ http://discover.nci.nih.gov/
} 


\section{Gene Expression Data}

The gene expression data were generated by Scherf et al. (2) in the same study that reported the drug response profiles described in §2.3. Cell collection and mRNA purification were described in their published report (2). The investigators used cDNA microarrays technology to quantify gene expression levels (2, 29). Expression profiles of 9,706 genes were assayed, and the data file is available on-line. ${ }^{7}$ The gene expression data is described in Table 4.1.

Table 4. 1: Gene Expression Data Description

\begin{tabular}{|l|l|}
\hline Column A: & IMAGE Clone ID \\
\hline Column B: & Gene description \\
\hline Column C: & $\begin{array}{l}\text { 5ACC: 5' genbank accession number (5' genbank is database of } \\
5 ' \text { untranslated regions of eukaryotic mRNAs. 5-UTR } \\
\text { sequences were defined as the mRNA region spanning from the } \\
\text { cap site to the starting codon (excluded)) }\end{array}$ \\
\hline Column D: & $\begin{array}{l}\text { 3ACC: } 3 \text { ' genbank accession number (3' genbank is the database } \\
\text { of 3' untranslated regions of eukaryotic mRNAs. 3'-UTR } \\
\text { sequences were defined as the mRNA region spanning from the } \\
\text { stop codon (excluded) to the poly (A)-addition site.) }\end{array}$ \\
\hline Column E-BL: & $\begin{array}{l}\text { Gene expression levels expressed as Log } 2 \text { (ratio), where ratio = } \\
\text { the red/green fluorescence ratio after computational balancing of } \\
\text { the two channels. }\end{array}$ \\
\hline
\end{tabular}

${ }^{7}$ http://discover.nci.nih.gov/datasetsNature2000.jsp 


\subsection{Descriptions of the Experiment}

\subsubsection{Data Preprocessing}

\section{Gene Screening and Identification}

The original gene profiling data measured the expression of 9,706 genes in NCI-60 lines (§2.3). For data quality control, the genes that were absent in more than four of the 60 cell lines were removed. There were a total of 1,374 genes remaining for the further analysis. All 1,374 genes showed a strong pattern of variation across the 60 cancer cell lines. The missing values may be the result of insufficient resolution, image corruption, dust, scratches on the slides, etc. Only the IMAGE Clone ID was provided for each gene in the original data. We used MatchMiner (30) to translate Clone ID into the gene symbols. MatchMiner identifies a gene by translating between the following different IDs: IMAGE and FISH clones, GenBank accession numbers, and UniGene cluster IDs.

\section{Missing Value Imputation}

Of the 1,374 remaining genes, some were absent in 4 or fewer of the 60 cell lines. For these, we used a nearest neighbor method (31) to impute the missing values. For example, suppose gene $g$ has missing values in cell line $i$, the algorithm used the weighted average of $k$ nearest genes' values in cell line $i$ to replace this missing value. In the literature, the imputation results were found to be stable and accurate for $k=10$ to 20 neighbors (32). In our experiments, we tried $k$ from 1 to 20 and found that the replaced missing values became stable when $\mathrm{k}$ was greater than or equal to 11 . By default, the number of neighbors is defined to be $0.01 *$ (number of features), so we used $k=13$ to implement the 
imputation of missing values. Correlation was used as the similarity metric to search for the neighbors. The $E M V$ package in software $R$ was employed to replace missing values.

\section{Defining Drug Sensitivity and Resistance}

We used the same cutoffs as described in §3.4.1 to define drug response. Specifically, for each drug, $\log _{10}\left(\mathrm{GI}_{50}\right)$ values were normalized across the 60 cell lines. For each drug, cell lines with $\log _{10}\left(\mathrm{GI}_{50}\right)$ values $0.5 \mathrm{SDs}$ above the mean were defined as resistant to this drug. Those with $\log _{10}\left(\mathrm{GI}_{50}\right)$ values $0.5 \mathrm{SDs}$ below the mean were defined as sensitive. And the remaining cell lines were defined as intermediate.

\subsubsection{Selecting Gene and Protein Predictors}

Gene expression data contains many irrelevant, noisy, and redundant features. Therefore, proper feature selection is even more essential in this part of the study.

Feature selection was performed with the varSelRF package in software $R$. We performed the feature selection and then model construction in two ways. In Method I, for each drug, the gene expression profiles and protein expression profiles in the NCI-60 panel were first integrated into one file. Then, the feature selection was performed on this integrated data file. Based on the identified predictors, a chemosensitivity classifier was constructed using the random forests algorithm. In Method II, for each drug, feature selection was first performed on the gene expression profiles and the protein expression profiles separately. Then, the top gene features and top protein features were aggregated in a stepwise manner to construct the chemosensitivity classifier. Details are provided as 
below.

In the NCI-60 gene expression data file, there are $G$ genes across $n$ cell lines. We notated the gene data file by a $G \times n$ matrix $X=\left(x_{g i}\right)$, where $x_{g i}$ denotes the expression measure of gene $g$ in cell line $i$. $X$ has dimension 1,374 x 60 after data pre-processing. We used a similar notation for the protein expression data. $P$ proteins across $n$ cell lines can be denoted as a $P$ x $n$ matrix $Y=\left(y_{p i}\right)$, where $y_{p i}$ is the expression measure of protein $p$ in cell line $i . Y$ has dimension 52 x 60.

Each cell line had a class label with three values (sensitive, intermediate, or resistant). We integrated both gene and protein expression into the model. Thus, each cell line had an integrated gene-protein expression profile $\boldsymbol{e}_{i}=\left(x_{1 i}, \ldots, x_{G i}, y_{1 i}, \ldots, y_{P i}\right)^{\prime}$ and a class label corresponding to a drug response.

\section{Method I. Aggregation of the proteomic and genomic data prior to feature selection}

For each drug, a new dataset was first created by integrating the gene expression profile and protein profile. Therefore, the new dataset contained 1,426 predictive variables (including 52 proteins, 1,374 mRNAs) and 1 drug response variable. Then, feature selection was performed on the aggregated profiles to identify gene and protein signatures. A chemosensitivity classifier was built upon these signatures. This method is outlined in Figure 4.2. Initially, the random forests algorithm ranked all the predictive variables and constructed a classifier based on all variables. Then, the least 20\% important variables were removed and a new classifier was constructed based on the remaining features. The out-of-bag error rate in bootstrap evaluation and the features list 
were recorded. This procedure was repeated until two variables remained in the dataset. Finally, the feature subset with the smallest error rate was selected. Here, the out-of-bag error rate in bootstrap evaluation was used as a feature selection criterion instead of an accuracy measure of model performance.

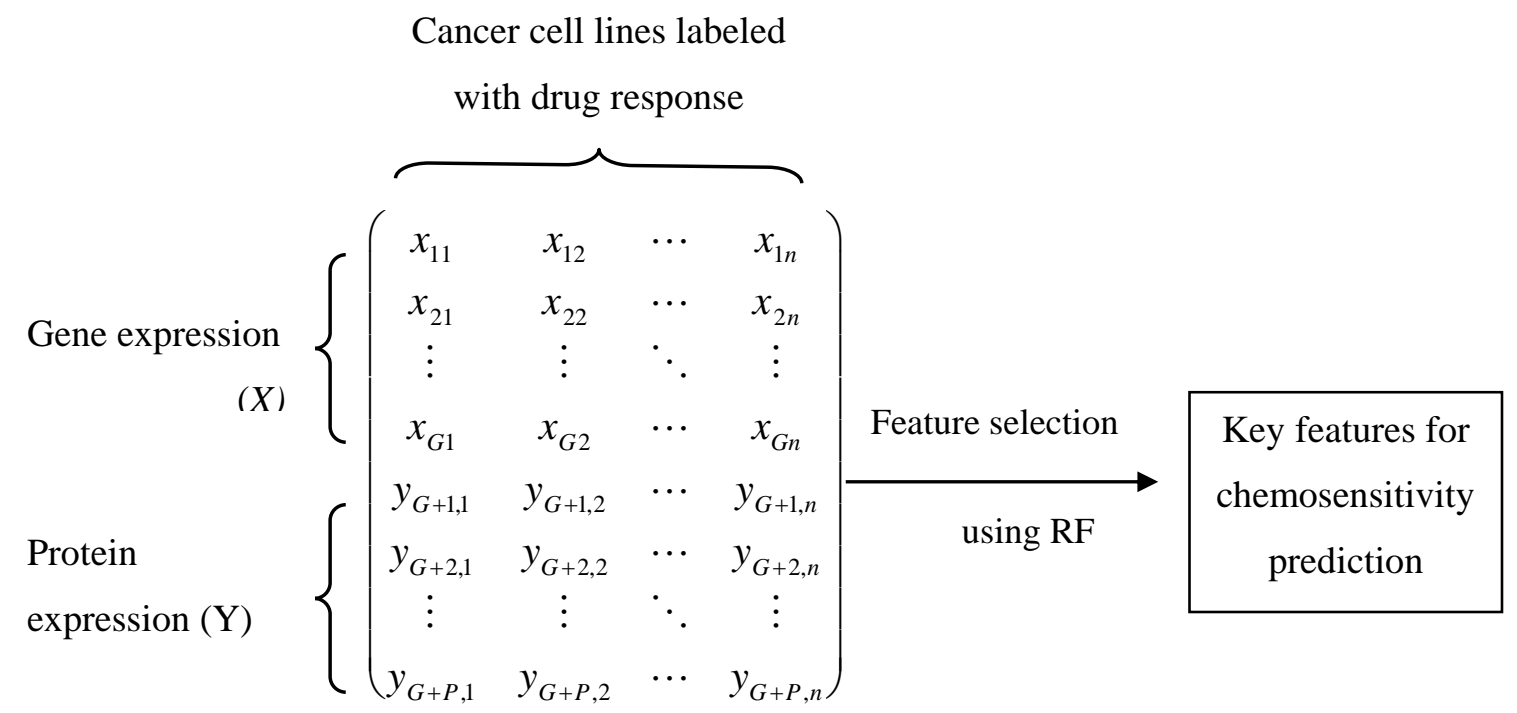

Figure 4. 2: Drug response prediction using proteomic and genomic profiling by method I

\section{Method II. Aggregation of the top-ranked proteins and genes from independent evaluation}

In Method II, gene and protein signatures were selected using the following three steps. First, the protein signatures were selected. The least important protein variables were removed in a stepwise manner. The subset of protein variables with the smallest out-of-bag error rate in bootstrap evaluation was selected. Second, gene signatures were selected. The same process as that employed in selecting protein signatures was employed. Third, the protein signatures were added to the gene signatures one by one from the most important to the least important. A chemosensitivity classifier was constructed for each of the mixed signatures. The mixed signatures and the prediction 
accuracy of each classifier were recorded. If protein signatures could not improve the prediction accuracy, then the optimal feature set was the gene signatures alone. Finally, the feature subset with the smallest error rate was selected. This method is outlined in Figure 4.3.

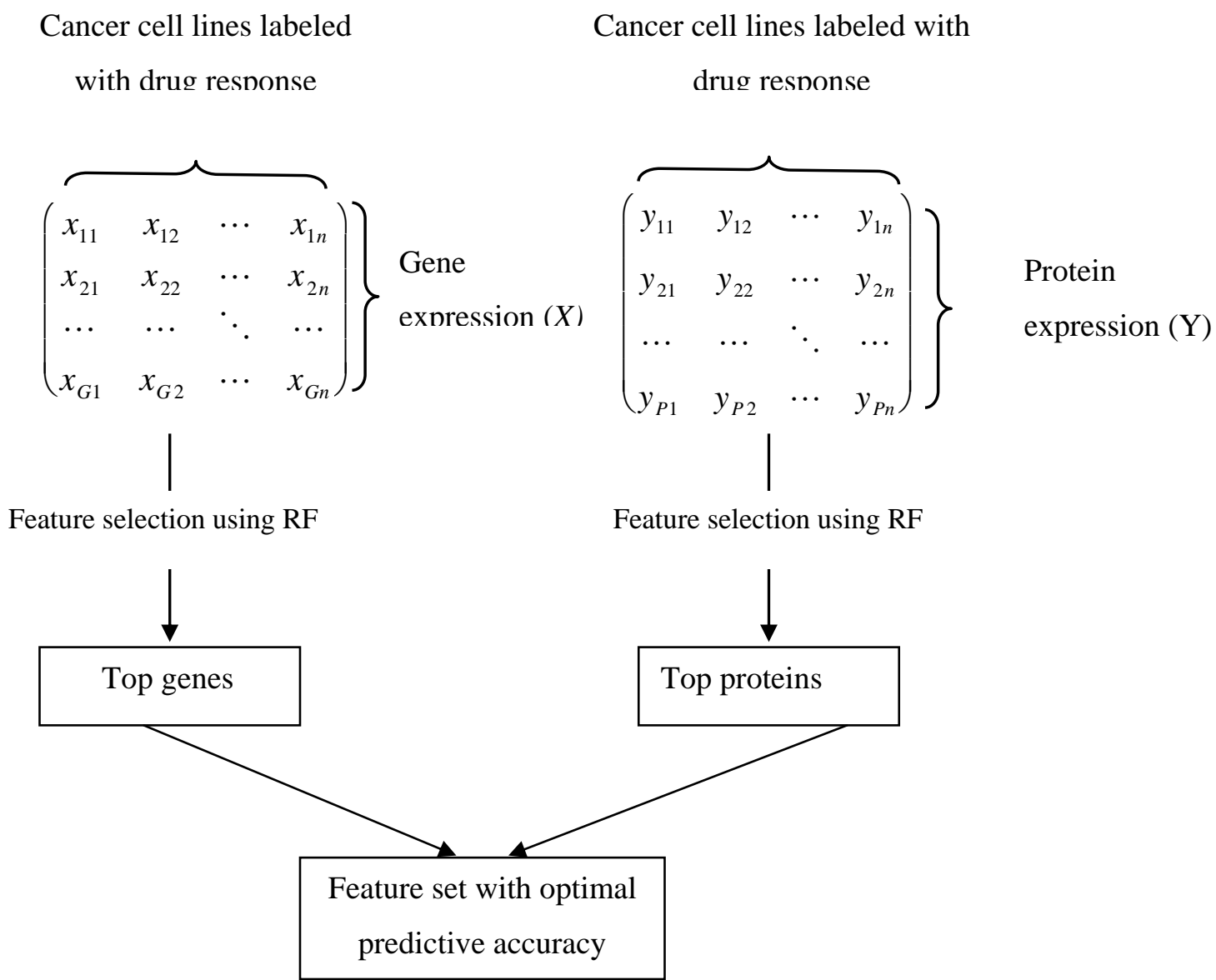

Figure 4. 3: Drug response prediction using proteomic and genomic profiling by method II 


\subsubsection{Classifying Drug Response of Individual Cell Lines}

After identifying the gene and protein signature, we constructed chemosensitivity classifiers using the random forests algorithm (§2.2.1) in software R. The out-of-bag error (§2.2.1) in bootstrap evaluation generated by the random forests algorithm was used as an accuracy measure. As discussed in §2.2.1, out-of-bag error is unbiased, so there is no need to perform cross-validation or use a separate dataset to evaluate the results (14).

\subsection{Results}

The chemosensitivity prediction accuracy for all evaluated drugs by using method I is shown in Figure 4.4, and those by using method II in Figure 4.5.

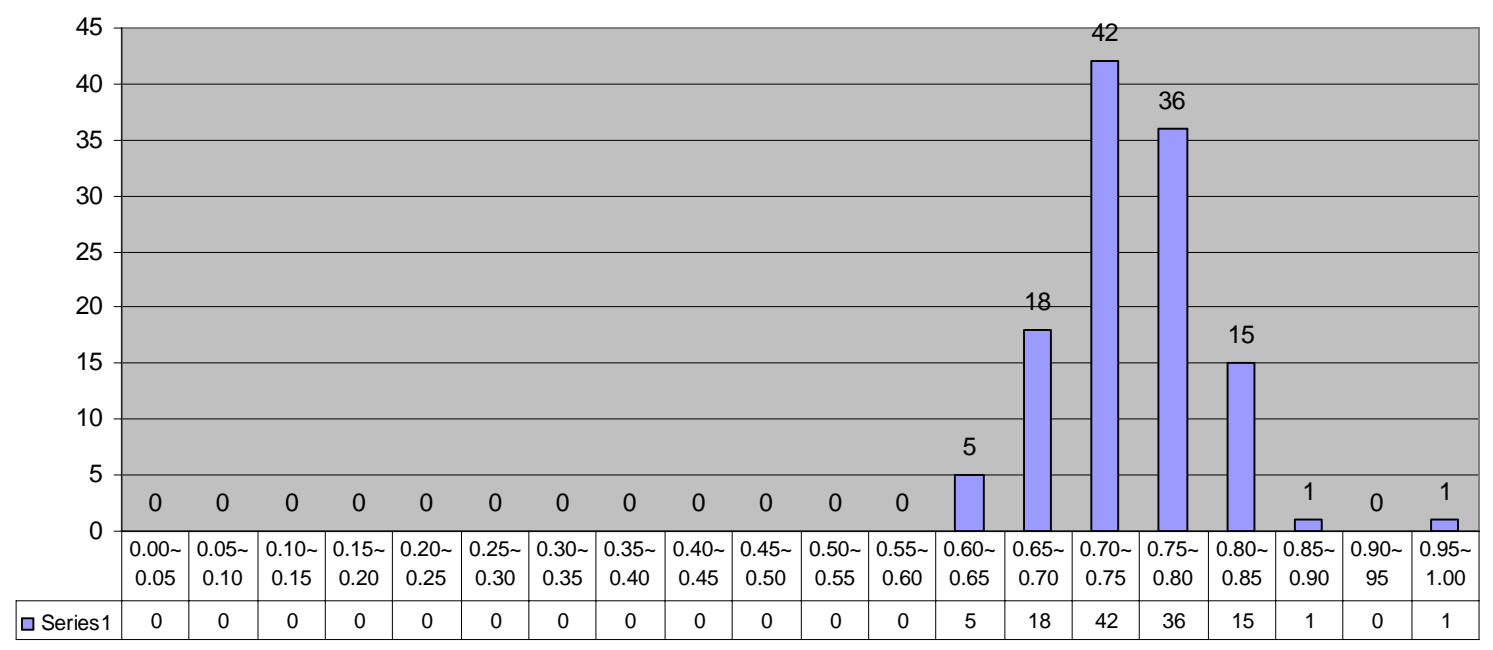

Figure 4. 4: Prediction accuracy of the constructed classifiers by method I 


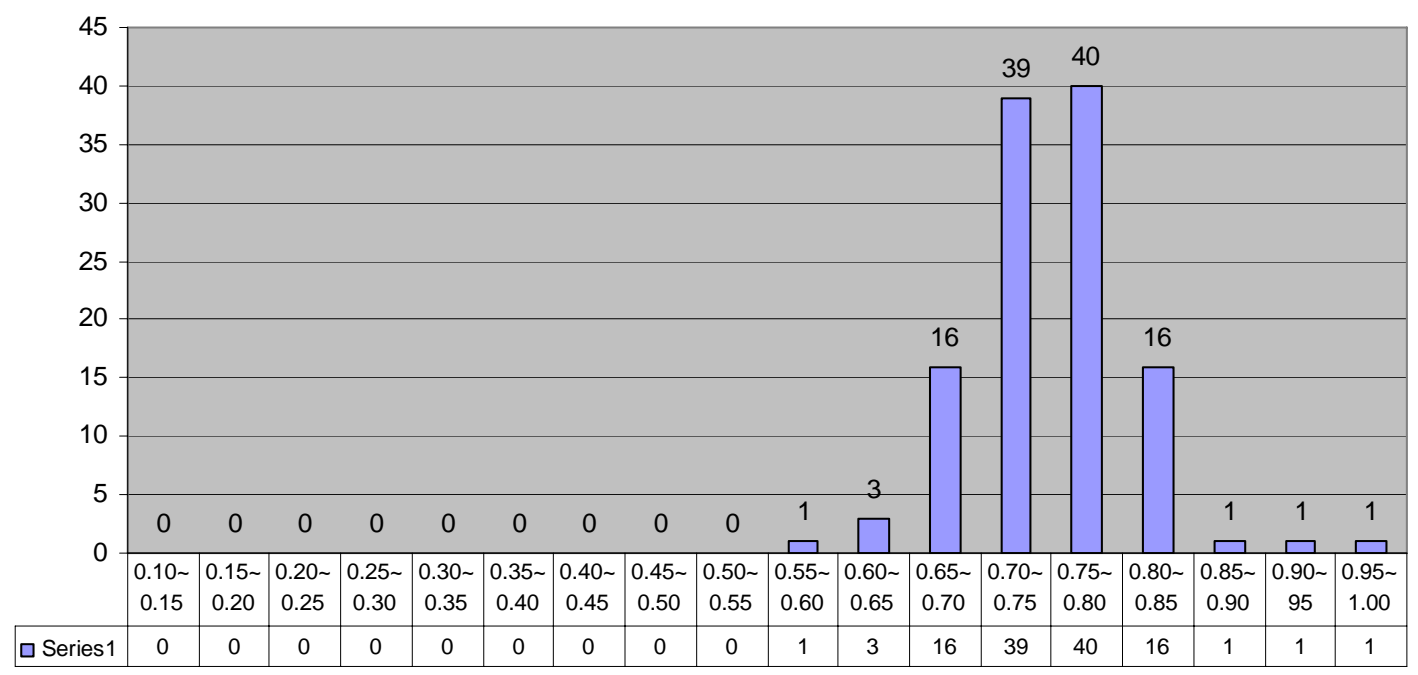

Figure 4. 5: Prediction accuracy of the constructed classifiers by method II

Both methods have certain advantages in constructing the chemosensitivity classifiers for the evaluated drugs. Therefore, we chose the better results from both methods as our final results. Those results are illustrated in Figure 4.6. Detailed results are provided in the appendix A.

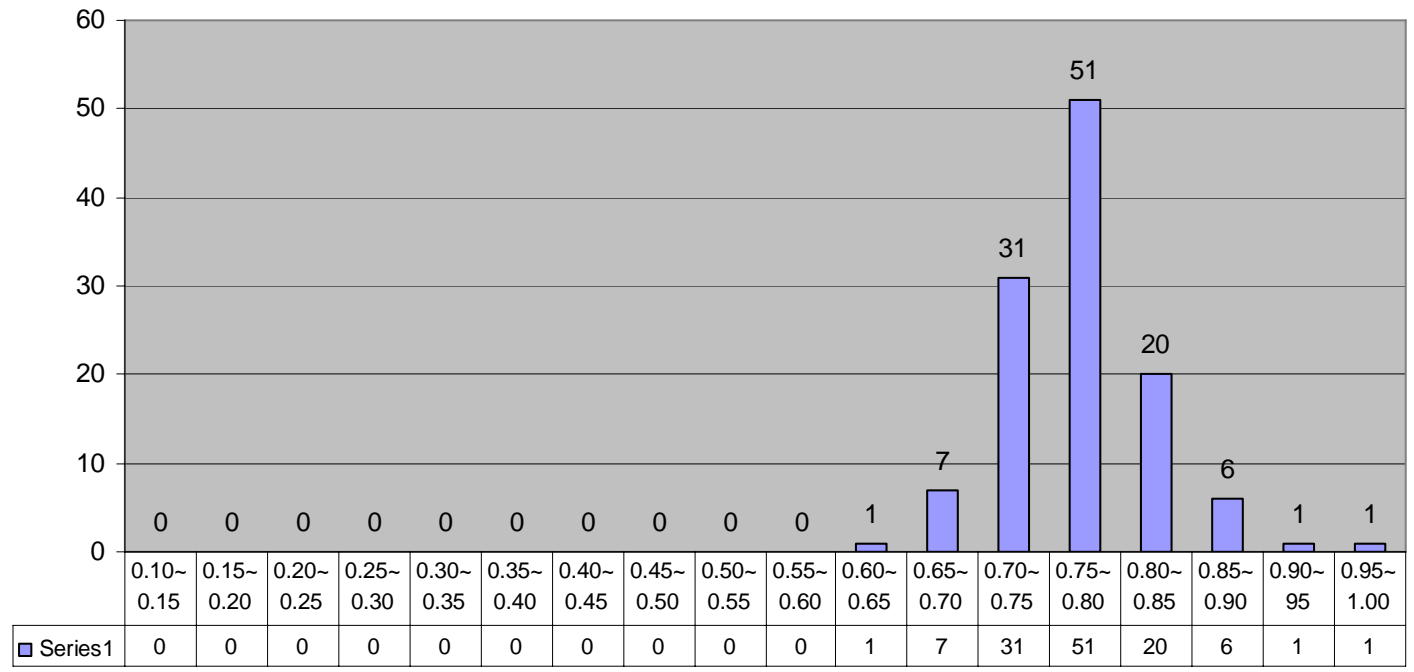

Figure 4. 6: Final prediction accuracy of the constructed optimal classifiers

To assess the significance of our prediction results based on the integrated profile 
(Figure 3.6), it was necessary to demonstrate that our prediction results were significantly better than random prediction. The same two methods as described in $§ 3.5$ were used to calculate the $P$ value. For all 118 drugs, we obtained $P$-values of zero. Therefore, our chemosensitivity classifiers were significantly better than random prediction $(P<0.001)$.

We also compared our results with those obtained by protein-based classifiers alone to further evaluate the improvement of these prediction results. Figure 4.7 shows the distribution of $P$-values from our evaluation. Seventy-six of the 118 integrated expression-based classifiers significantly $(P<0.05)$ improved the chemosensitivity prediction accuracy acquired by protein expression-based classifiers alone.

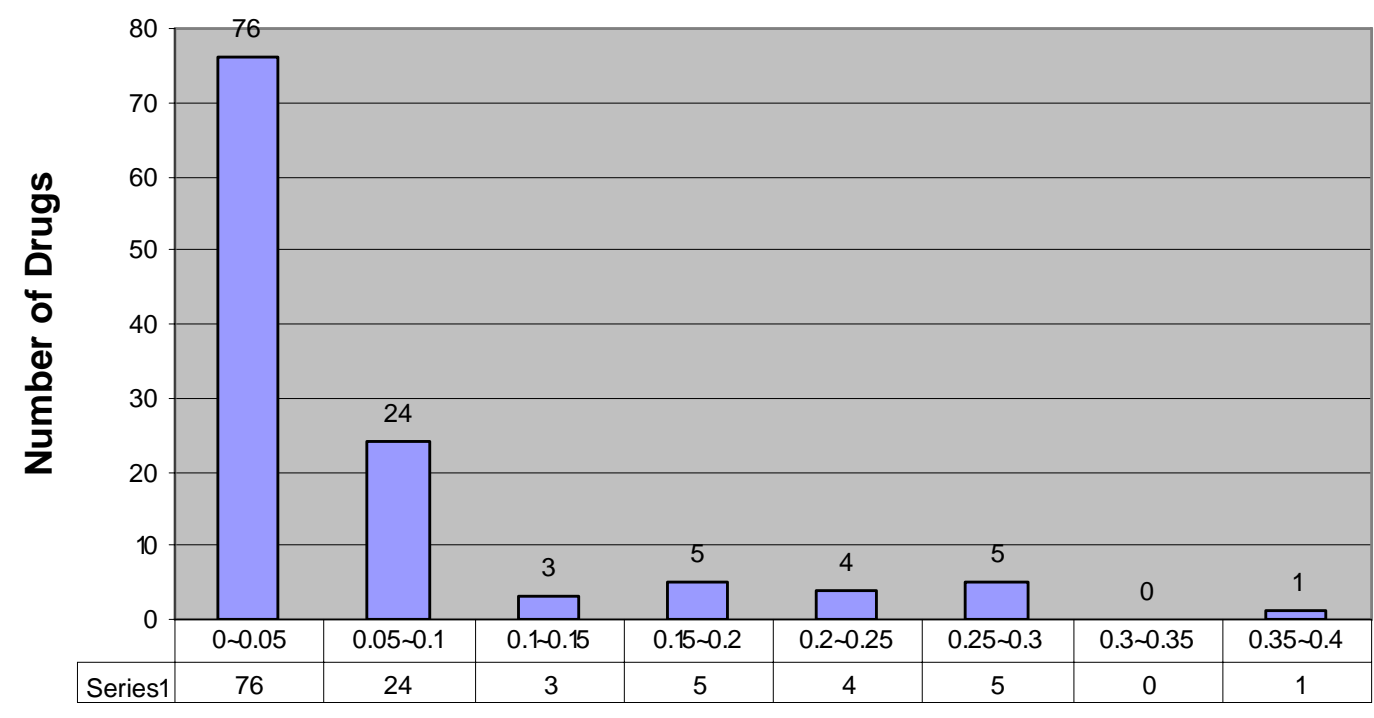

P-Value

Figure 4. 7: Improvement of the integrated molecular chemosensitivity classifiers over protein expression-based classifiers 


\subsection{Discussion}

With the development of high-throughput technologies, it is possible to quickly and efficiently acquire molecular expression data. Thus, new computational approaches are needed to integrate these diverse data and build multi-leveled biological models to predict chemosensitivity. Previous integrated analyses have focused on the correlation between mRNA and protein expression patterns. In this study, we developed a novel feature selection algorithm to identify gene and protein expression signatures to predict chemosensitivity. This scheme is a general approach to systematically evaluate genome-wide DNA, RNA, and protein contributions in cancer progression and drug sensitivity.

A particular challenge of integrated gene-protein expression-based chemosensitivity prediction is unbalanced data sets. The number of available features in the proteomic data (52 proteins) is much lower than that in the transcriptional data (1,374 genes). These unbalanced data sets make it difficult to construct integrated gene-protein expression-based chemosensitivity classifiers. Here, we developed two stepwise feature selection schemes to account for this problem. In Method I, the gene expression and protein expression profiles were first combined into one data file, and then, the feature selection was performed on the integrated file to identify the optimal chemosensitivity signatures. Based on the identified predictors, a chemosensitivity classifier was constructed using the random forests algorithm. In Method II, the feature selection was performed on the gene expression and protein expression profiles separately, and then, the identified top gene subset and top protein subset were integrated in a stepwise manner 
to build the classifier. The optimal classifiers built from both approaches were selected as the final results.

\subsection{Summary}

In the previous chapter, we described how we built a machine learning model system to predict chemosensitivity exclusively based on proteomic profiling. In this chapter, we described how we developed a novel algorithm to integrate the genomic profiling into our previous model and constructed chemosensitivity classifiers based on the integrating genomic and proteomic profiling. The classification accuracy of all the evaluated 118 chemotherapeutic agents was remarkably better $(P<0.001)$ than what would be achieved by chance. Furthermore, 76 out of the 118 integrated genomic and proteomic classifiers for chemosensitivity significantly $(P<0.05)$ improved the accuracy of protein expression-based classifiers alone. Our results showed that the multi-leveled gene and protein expression signatures could significantly increased the accuracy of drug response prediction. 


\section{Chapter 5}

\section{Contributions and Discussions}

\subsection{Contributions}

Accurately predicting an individual patient's response to anticancer drugs would allow for the design of more effective treatments. Recent studies in this area have focused on transcriptional profiling. However, protein ultimately plays an essential role in the cancer development and progression. So predicting chemosensitivity based on proteomic profiling may yield more direct answers to functional and pharmacologic questions. Furthermore, integrating proteomic and genomic profiling may reveal important information of how cancer develops and progresses from a multi-leveled approach. Previous studies that integrated protein and genomic profiles have focused on the correlation between gene and protein expression patterns and may have potentially omitted some important biological information regarding chemosensitivity mechanisms.

Our study resolved two challenges in these research areas. Previous attempts to predict chemosensitivity based solely on protein profiles have been limited by the small amount of available protein expression data resulting from difficulties inherent in current proteomics technology. In the present study, we developed a machine learning model that allowed chemosensitivity classifiers based on proteomic signatures alone to accurately predict drug response. This is the first study to accurately $(P<0.02)$ predict cell line chemosensitivity exclusively based on proteomic profiling. Furthermore, we extended our model by integrating genomic profiling with proteomic profiling and constructing chemosensitivity classifiers based on the integrated profiles. Seventy-six out of the 118 
classifiers could significantly $(P<0.05)$ improve the chemosensitivity prediction accuracy acquired by protein expression-based classifiers. These results indicate that our integrated genomic and proteomic approach could further increase chemosensitivity prediction accuracy.

\subsection{Discussions}

Most machine learning algorithms were first developed from and applied in the domains of banking, marketing, and business. The data in these domains are usually characterized by large sample sizes and a small number of features. However, the data in the current study, typical of bioinformatics data, contained a small number of samples but a large number of features. As a result, there is a lot of irrelevant, noisy, and redundant information in these data. Therefore, it was not clear whether these existing machine learning algorithms would be suitable for our integrated proteomic and genomic profiling of untreated cell lines.

In addition, different datasets have different characteristics. There is no algorithm that has superior performance for all applications. In the future, other machine learning algorithms, such as Naive Bayes, JRip, J48 and OneR, will be applied in chemosensitivity prediction using 118 drug data. The evaluation of various algorithms will help in indentifying appropriate method for a new data application. 


\section{Appendix A: Detailed Results of the Integrated Gene-Protein Expression-Based Chemosensitivity Classifiers}

Table A. 1 Number of Selected Features in the Integrated Gene-Protein Expression-Based Chemosensitivity Classifiers

\begin{tabular}{|c|c|c|c|c|c|c|}
\hline \# & Drug & NSC & Approach & $\begin{array}{l}\text { Total \# of } \\
\text { Features }\end{array}$ & $\begin{array}{l}\text { \# of } \\
\text { Selected } \\
\text { Genes }\end{array}$ & $\begin{array}{c}\text { \# of } \\
\text { Selected } \\
\text { Proteins }\end{array}$ \\
\hline 1 & Mitomycin & 26980 & 1 & 11 & 9 & 2 \\
\hline 2 & Porfiromycin & 56410 & 1 & 32 & 30 & 2 \\
\hline 3 & Carmustine (BCNU) & 409962 & 1 & 14 & 13 & 1 \\
\hline 4 & Chlorozotocin & 178248 & 2 & 13 & 13 & 0 \\
\hline 5 & Clomesone & 338947 & 2 & 5 & 5 & 0 \\
\hline 6 & Lomustine (CCNU) & 79037 & 2 & 6 & 6 & 0 \\
\hline 7 & Mitozolamide & 353451 & 2 & 11 & 10 & 1 \\
\hline 8 & PCNU & 95466 & 1 & 14 & 13 & 1 \\
\hline 9 & $\begin{array}{l}\text { Semustine } \\
\text { (MeCCNU) }\end{array}$ & 95441 & 1 & 9 & 8 & 1 \\
\hline 10 & Asaley & 167780 & 2 & 11 & 11 & 0 \\
\hline 11 & Busulfan & 750 & 2 & 4 & 4 & 0 \\
\hline 12 & Carboplatin & 241240 & 1 & 21 & 21 & 0 \\
\hline 13 & Chlorambucil & 3088 & 2 & 7 & 7 & 0 \\
\hline 14 & Cisplatin & 119875 & 2 & 7 & 6 & 1 \\
\hline 15 & Cyclodisone & 348948 & 2 & 10 & 10 & 0 \\
\hline 16 & $\begin{array}{l}\text { Diaminocyclohexyl- } \\
\text { Pt-II }\end{array}$ & 271674 & 2 & 26 & 26 & 0 \\
\hline 17 & Dianhydrogalactitol & 132313 & 2 & 9 & 9 & 0 \\
\hline
\end{tabular}




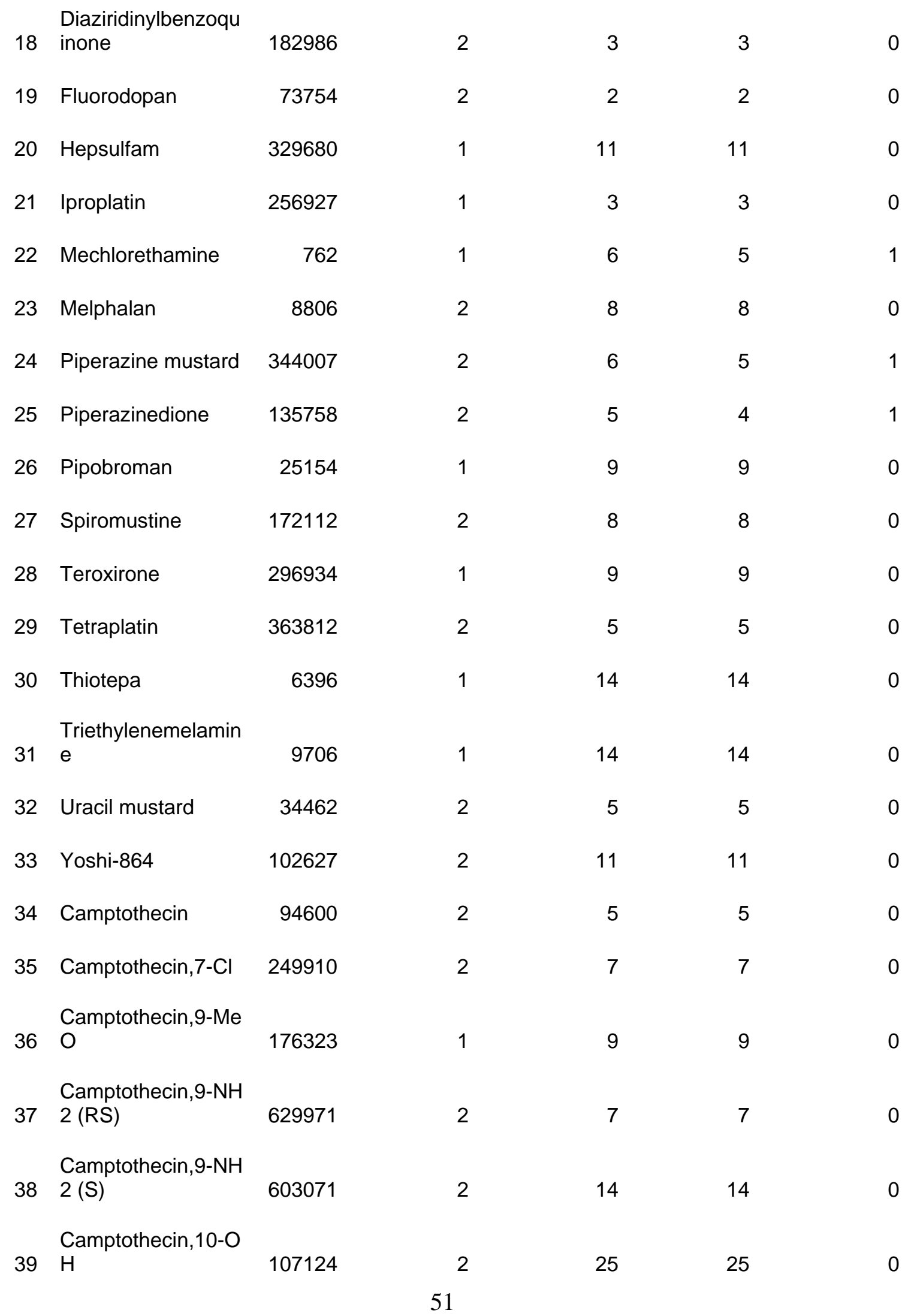




\begin{tabular}{|c|c|c|c|c|c|c|}
\hline 40 & $\begin{array}{l}\text { Camptothecin,11-fo } \\
\text { rmyl (RS) }\end{array}$ & 606172 & 1 & 5 & 5 & 0 \\
\hline 41 & $\begin{array}{l}\text { Camptothecin,11-H } \\
\text { OMe (RS) }\end{array}$ & 606173 & 1 & 14 & 14 & 0 \\
\hline & Camptothecin,20 & 60649 & & & & \\
\hline 42 & -ester (S) & 7 & 2 & 14 & 14 & 0 \\
\hline 43 & $\begin{array}{l}\text { Camptothecin,20-e } \\
\text { ster (S) }\end{array}$ & 606985 & 1 & 14 & 14 & 0 \\
\hline 44 & $\begin{array}{l}\text { Camptothecin,20-e } \\
\text { ster (S) }\end{array}$ & 610456 & 2 & 6 & 6 & 0 \\
\hline 45 & $\begin{array}{l}\text { Camptothecin,20-e } \\
\text { ster (S) }\end{array}$ & 618939 & 2 & 5 & 4 & 1 \\
\hline 46 & AmoOfide & 308847 & 2 & 9 & 9 & 0 \\
\hline 47 & Amsacrine & 249992 & 1 & 9 & 9 & 0 \\
\hline 48 & $\begin{array}{l}\text { Anthrapyrazole-deri } \\
\text { vative }\end{array}$ & 355644 & 2 & 4 & 4 & 0 \\
\hline 49 & Bisantrene & 337766 & 2 & 4 & 4 & 0 \\
\hline 50 & Daunorubicin & 82151 & 1 & 4 & 4 & 0 \\
\hline 51 & Deoxydoxorubicin & 267469 & 2 & 4 & 4 & 0 \\
\hline 52 & Doxorubicin & 123127 & 2 & 4 & 4 & 0 \\
\hline 53 & Etoposide & 141540 & 2 & 3 & 3 & 0 \\
\hline 54 & Menogaril & 269148 & 2 & 14 & 14 & 0 \\
\hline 55 & Mitoxantrone & 301739 & 1 & 11 & 10 & 1 \\
\hline 56 & $\begin{array}{l}\text { Oxanthrazole } \\
\text { (piroxantrone) }\end{array}$ & 349174 & 2 & 16 & 16 & 0 \\
\hline 57 & Teniposide & 122819 & 2 & 8 & 8 & 0 \\
\hline 58 & $\begin{array}{l}\text { Zorubicin } \\
\text { (Rubidazone) }\end{array}$ & 164011 & 2 & 6 & 6 & 0 \\
\hline
\end{tabular}



59 L-AsparagiOse
Cyanomorpholinod
60 oxorubicin

109229

357704

142982

354646

1

26

22

4

$\mathrm{N}-\mathrm{N}-$ Dibenzyl-daun

63

omycin

268242

366140

64 Pyrazoloacridine

5-6-Dihydro-5-azac

65 ytidine

alpha-2'-Deoxythio

66 guanosine

71851

102816

67 Azacytidine

beta-2'-Deoxythiog

68 uanosine

69 Thioguanine

70 Aminopterin

Aminopterin-derivat

71 ive

Aminopterin-derivat

72 ive

184692

623017

633713

74 an-antifol

Baker's-soluble-anti

75 folate

76 Methotrexate
139105

71261

752

132483

134033

2

1

2

2

1

740
1

14

4

26

25

1

2

2

13

12

1

1

0

0

$3 \quad 3 \quad 0$

4

0

0

$4 \quad 4 \quad 0$

73 an-antifol

5

0

$5 \quad 5$

53 


\begin{tabular}{|c|c|c|c|c|c|c|}
\hline 77 & $\begin{array}{l}\text { Methotrexate-deriv } \\
\text { ative }\end{array}$ & 174121 & 2 & 3 & 3 & 0 \\
\hline 78 & Trimetrexate & 352122 & 1 & 7 & 6 & 1 \\
\hline 79 & Gua0zole & 1895 & 2 & 4 & 4 & 0 \\
\hline 80 & Hydroxyurea & 32065 & 1 & 3 & 3 & 0 \\
\hline 81 & Pyrazoloimidazole & 51143 & 1 & 26 & 25 & 1 \\
\hline 82 & Aphidicolin-glyciOte & 303812 & 1 & 17 & 17 & 0 \\
\hline 83 & Cyclocytidine & 145668 & 1 & 11 & 11 & 0 \\
\hline 84 & Cytarabine (araC) & 63878 & 1 & 17 & 17 & 0 \\
\hline 85 & Floxuridine (FUdR) & 27640 & 2 & 8 & 8 & 0 \\
\hline 86 & Fluorouracil (5FU) & 19893 & 2 & 8 & 6 & 2 \\
\hline 87 & Ftorafur & 148958 & 1 & 14 & 14 & 0 \\
\hline 88 & Thiopurine (6MP) & 755 & 1 & 9 & 8 & 1 \\
\hline 89 & Acivicin & 163501 & 2 & 5 & 5 & 0 \\
\hline 90 & $\begin{array}{l}\text { Dichloroallyl-lawson } \\
\text { e }\end{array}$ & 126771 & 1 & 3 & 3 & 0 \\
\hline 91 & DUP785 (brequiar) & 368390 & 1 & 4 & 4 & 0 \\
\hline 92 & L-Alanosine & 153353 & 1 & 9 & 8 & 1 \\
\hline 93 & $\begin{array}{l}\text { N-phosphonoacetyl } \\
\text {-L-aspartic-acid }\end{array}$ & 224131 & 1 & 6 & 5 & 1 \\
\hline 94 & Pyrazofurin & 143095 & 1 & 7 & 7 & 0 \\
\hline 95 & Colchicine & 757 & 1 & 7 & 6 & 1 \\
\hline 96 & $\begin{array}{l}\text { Colchicine-derivativ } \\
\text { e }\end{array}$ & 33410 & 2 & 4 & 3 & 1 \\
\hline 97 & Dolastatin-10 & 376128 & 1 & 14 & 12 & 2 \\
\hline
\end{tabular}




\begin{tabular}{|c|c|c|c|c|c|c|}
\hline 98 & Halichondrin B & 609395 & 2 & 4 & 4 & 0 \\
\hline 99 & Maytansine & 153858 & 1 & 3 & 3 & 0 \\
\hline 100 & Trityl-cysteine & 83265 & 1 & 14 & 14 & 0 \\
\hline 101 & Vinblastine-sulfate & 49842 & 2 & 3 & 3 & 0 \\
\hline 102 & Vincristine-sulfate & 67574 & 1 & 2 & 2 & 0 \\
\hline 103 & Taxol (Paclitaxel) & 125973 & 2 & 10 & 8 & 2 \\
\hline 104 & Taxol analog & 600222 & 2 & 6 & 6 & 0 \\
\hline 105 & Taxol analog & 656178 & 1 & 5 & 5 & 0 \\
\hline 106 & Taxol analog & 658831 & 2 & 8 & 8 & 0 \\
\hline 107 & Taxol analog & 661746 & 2 & 3 & 3 & 0 \\
\hline 108 & Taxol analog & 664402 & 2 & 5 & 5 & 0 \\
\hline 109 & Taxol analog & 664404 & 2 & 4 & 4 & 0 \\
\hline 110 & Taxol analog & 666608 & 2 & 8 & 8 & 0 \\
\hline 111 & Taxol analog & 671867 & 2 & 13 & 13 & 0 \\
\hline 112 & Taxol analog & 671870 & 2 & 4 & 4 & 0 \\
\hline 113 & Taxol analog & 673187 & 2 & 11 & 11 & 0 \\
\hline 114 & Taxol analog & 673188 & 2 & 6 & 5 & 1 \\
\hline 115 & Geldanamycin & 330500 & 1 & 11 & 11 & 0 \\
\hline & $\begin{array}{l}\text { 3-Hydropicolinaldeh } \\
\text { yde-thiosemicarbaz }\end{array}$ & & & & & \\
\hline 116 & one & 95678 & 2 & 17 & 17 & 0 \\
\hline & $\begin{array}{l}\text { 5-Hydroxypicolinald } \\
\text { ehyde-thiosemicarb }\end{array}$ & & & & & \\
\hline 117 & azone & 107392 & 2 & 25 & 24 & 1 \\
\hline 118 & $\begin{array}{l}\text { Inosine-glycodialde } \\
\text { hyde }\end{array}$ & 118994 & 1 & 11 & 11 & 0 \\
\hline
\end{tabular}


Table A. 2 Names of Selected Features in the Integrated Gene-Protein Expression-Based Chemosensitivity Classifiers

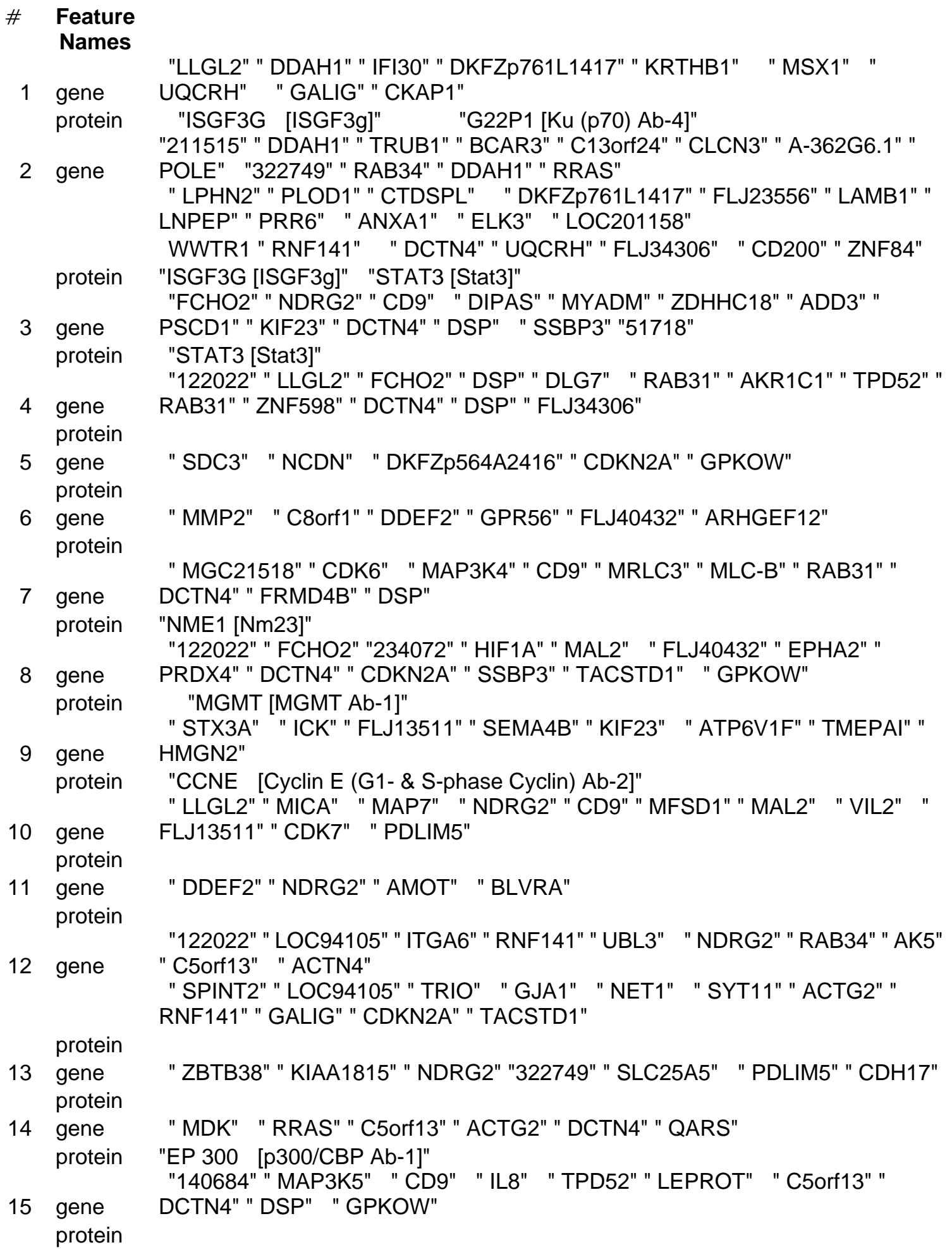




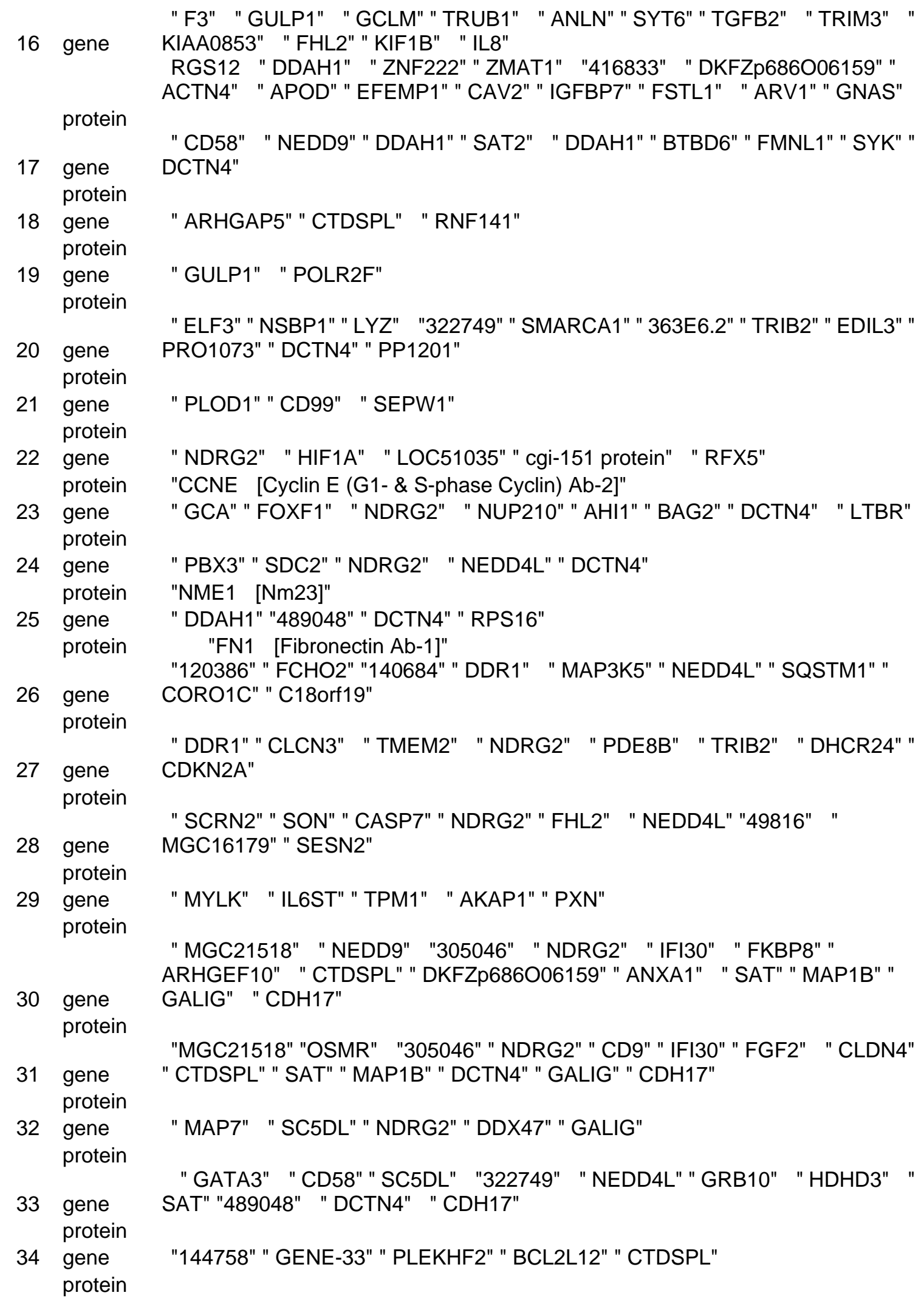




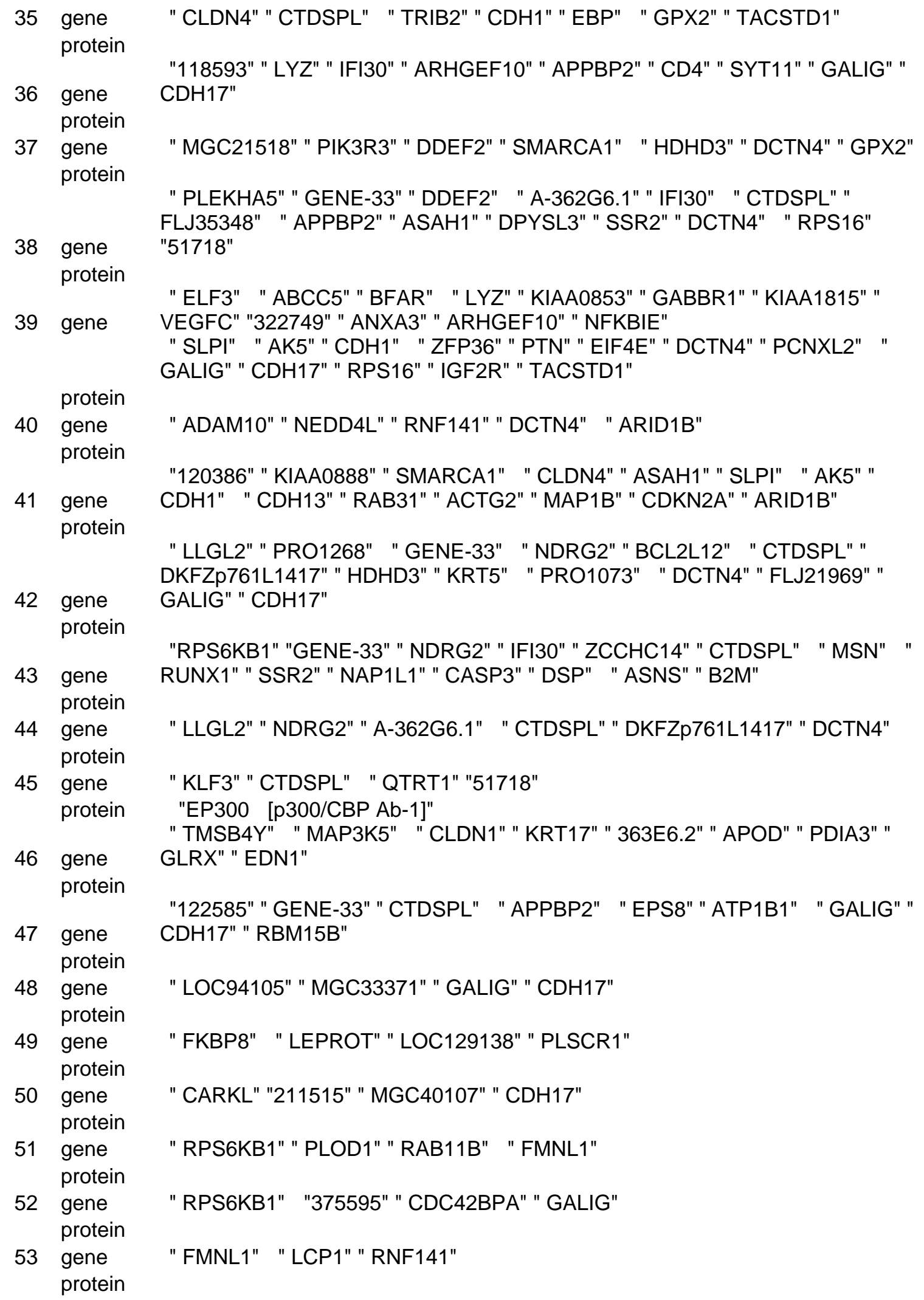




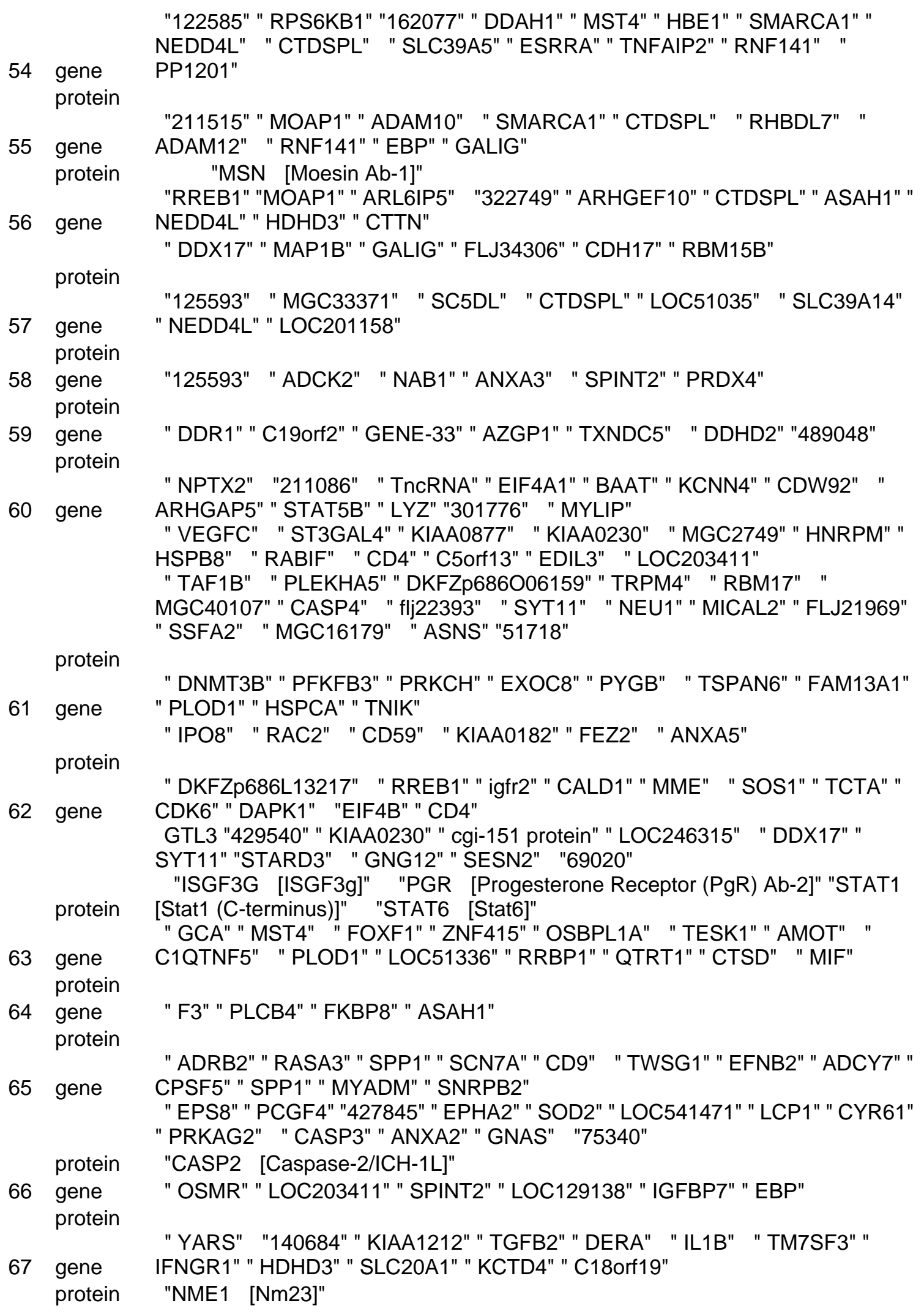




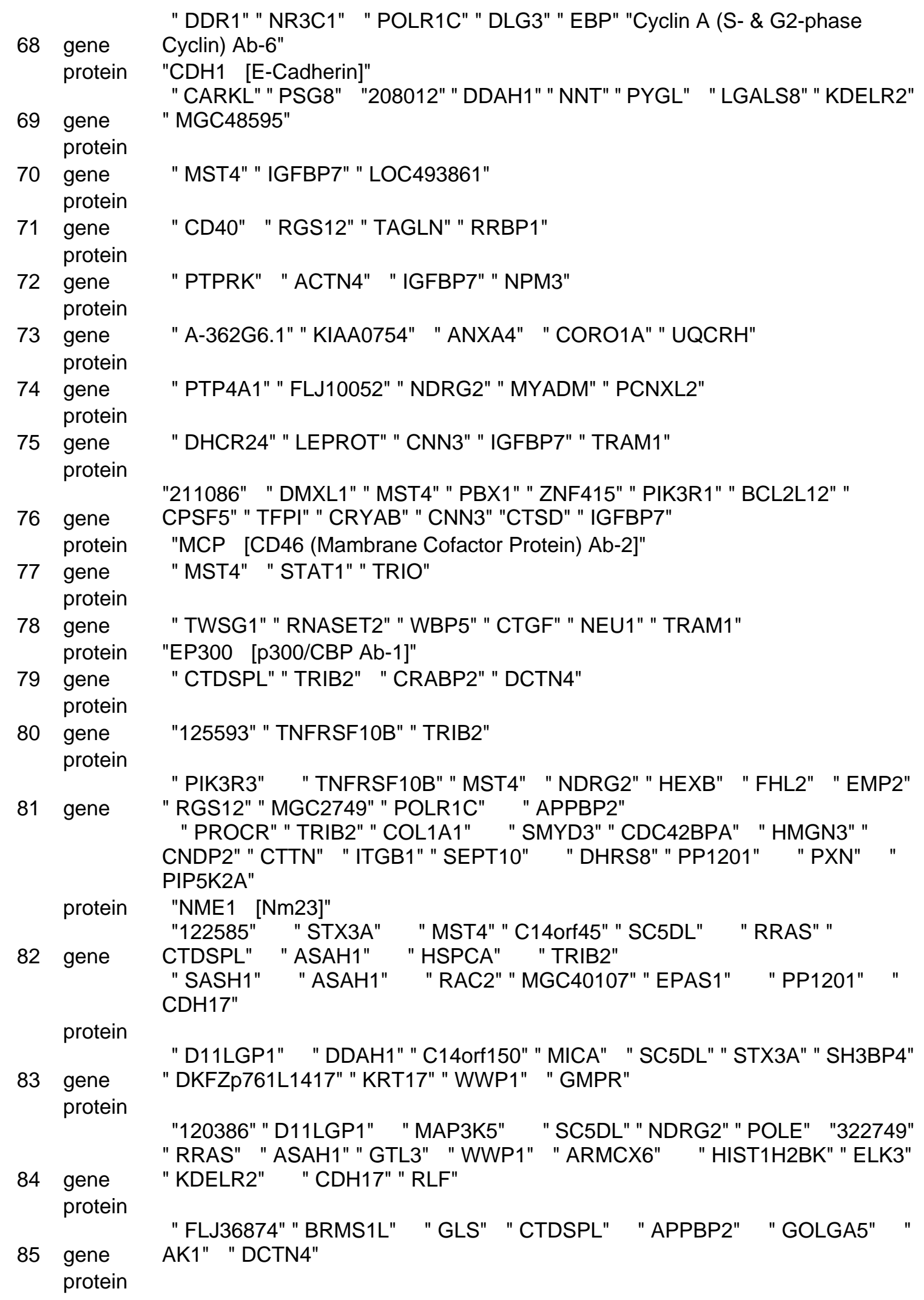




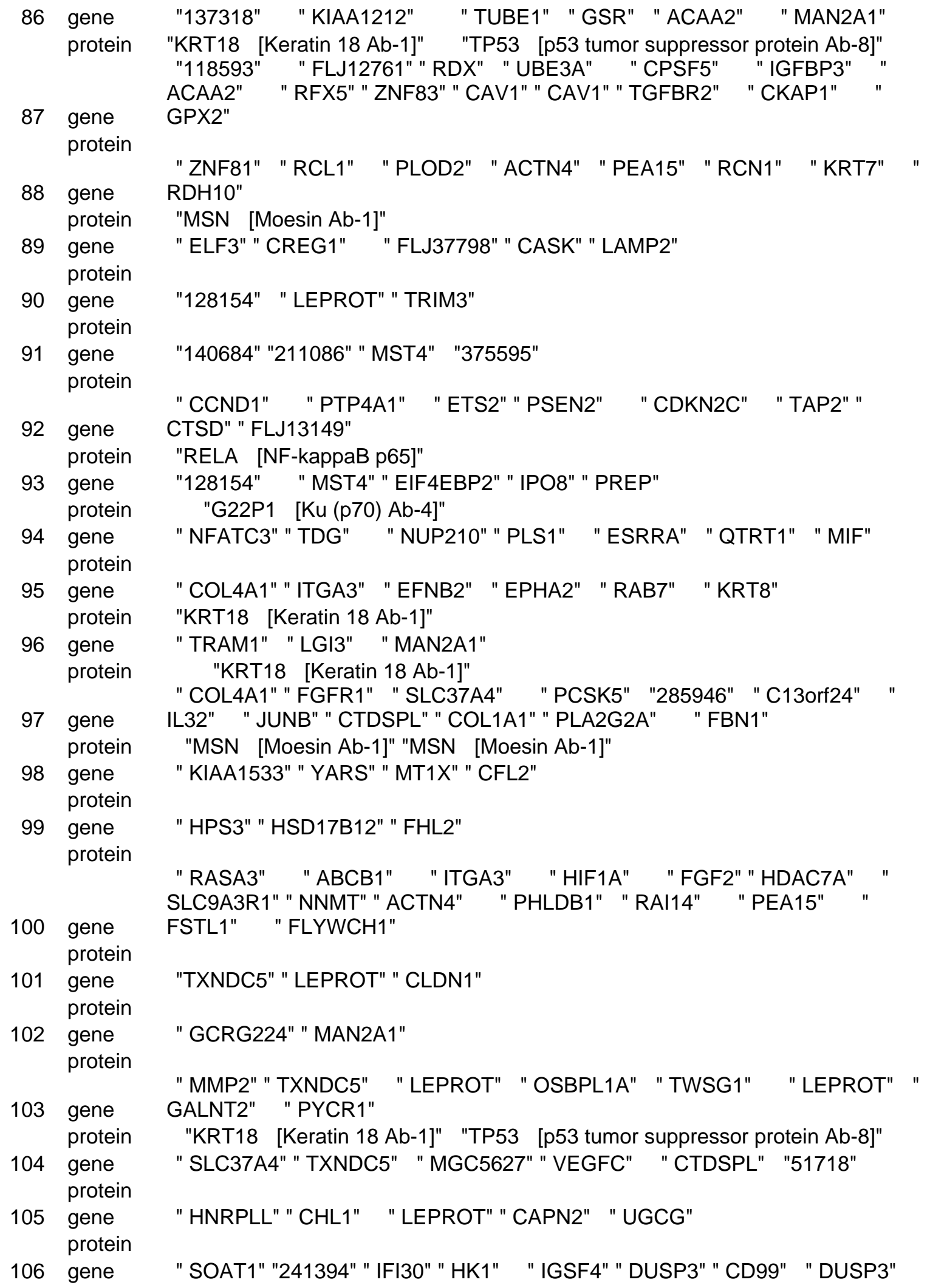




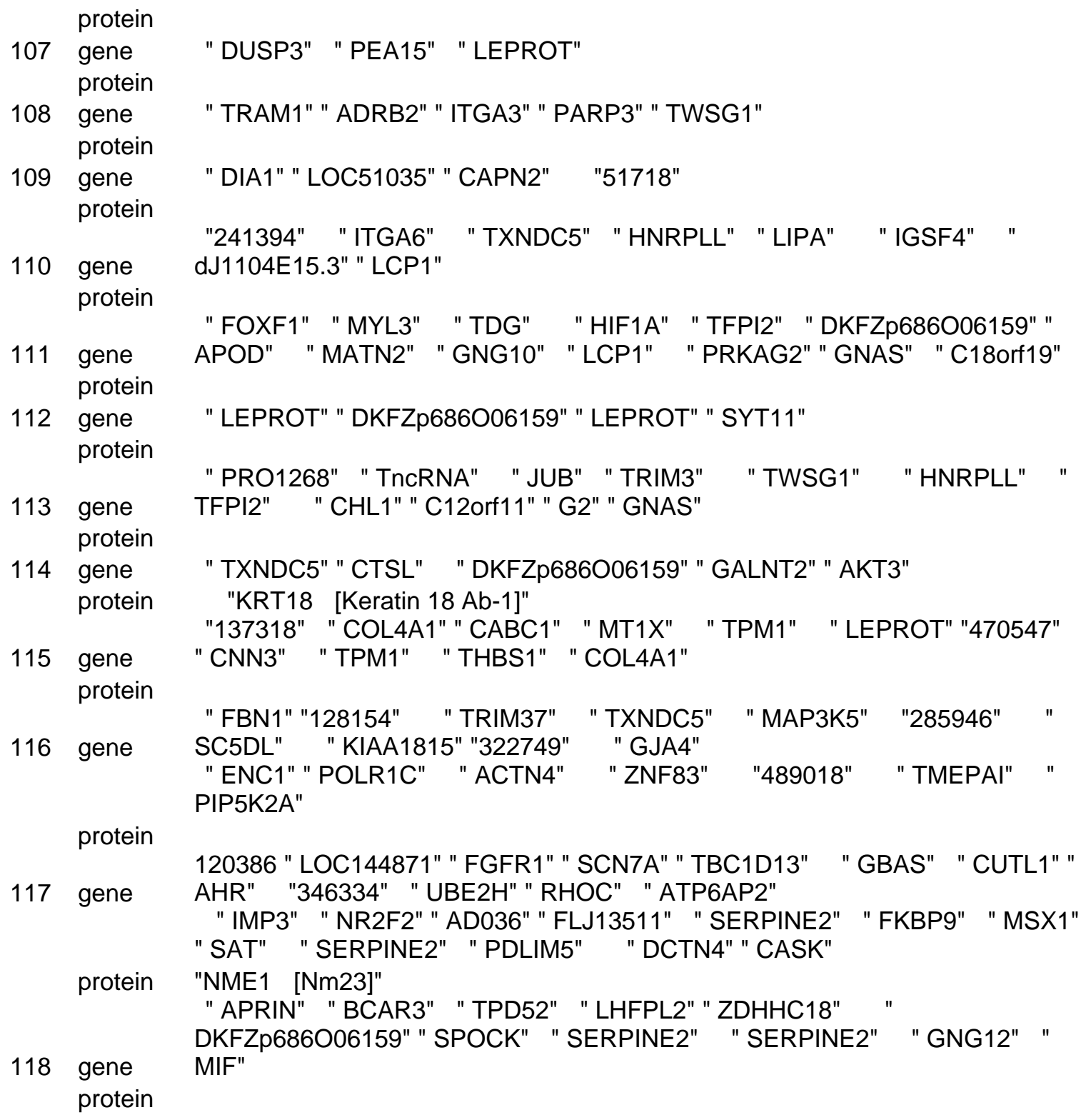




\section{Reference}

1. A.H. Bild, G. Yao, J.T. Chang, Q. Wang, A. Potti, D. Chasse et al., "Oncogenic Pathway Signatures in Human Cancers as a Guide to Targeted Therapies”, Nature, vol.439, pp.353-357, 2006

2. U. Scherf, D.T. Ross, M. Waltham, L.H. Smith, J.K. Lee , L. Tanabe et al., “A Gene Expression Database for the Molecular Pharmacology of Cancer”, Nature Genetics, vol.24, pp.236-244, 2000.

3. J.E. Staunton, D.K. Slonim, H.A. Coller, P. Tamayo, M.J. Angelo, J. Park et al., “Chemosensitivity Prediction by Transcriptional Profiling”, Proc.Natl.Acad.Sci.U.S.A, vol.98, pp.10787-10792, 2001.

4. G. Szakacs, J.P. Annereau, S. Lababidi, U. Shankavaram, A. Arciello, K.J. Bussey et al. "Predicting Drug Sensitivity and Resistance: Profiling ABC Transporter Genes in Cancer Cells”, Cancer Cell, vol.6, pp.129-137, 2004.

5. D.B. Solit, L.A. Garraway , C.A. Pratilas, A. Sawai, G. Getz, A. Basso et al., "BRAF Mutation Predicts Sensitivity to MEK Inhibition”, Nature, vol.439, pp.358-362, 2006.

6. S. Nishizuka, L. Charboneau, L. Young, S. Major, W.C. Reinhold, M. Waltham et al., "Proteomic Profiling of the NCI-60 Cancer Cell Lines using New High-Density Reverse-Phase Lysate Microarrays”, Proc.Natl.Acad.Sci.U.S.A, vol.100, pp.14229-14234, 2003.

7. G. Chen, T.G. Gharib, C.C. Huang, J.M. Taylor, D.E. Misek, S.L. Kardia et al., “Discordant Protein and mRNA Expression in Lung Adenocarcinomas”, Mol.Cell Proteomics., vol.1, pp.304-313, 2002.

8. S. Varambally, J. Yu, B. Laxman, D.R. Rhodes, R. Mehra, S.A. Tomlins et al., "Integrated Genomic and Proteomic Analysis of Prostate Cancer Reveals Signatures of Metastatic Progression”, Cancer Cell, vol.8, pp.393-406, 2005.

9. I.H. Witten, E. Frank. Data mining: Practical Machine Learning Tools and Techniques, 2nd ed. New York: Morgan Kaufmann, 2005.

10. http://www.cancer.gov/Templates/db_alpha.aspx?CdrID=372925, 2007.

11. G.Z.Acton, “Genomics Approaches to Improving Drug Treatments”, Medscape Pharmacotherapy vol.3, no.1, 2001.

12. R. Simon, "Roadmap for Developing and Validating Therapeutically Relevant Genomic Classifiers”, J Clin Oncol, vol.23, pp.7332-7341, 2005 
13. S.P. Gygi, B. Rist, S.A. Gerber, F. Turecek, M.H. Gelb, R. Aebersold, “Quantitative Analysis of Complex Protein Mixtures using Isotope-Coded Affinity Tags”,

Nat.Biotechnol., vol.17, pp.994-999, 1999.

14. L. Breiman, “Random Forests”, Machine Learning, vol.45, pp.5-32, 2001.

15. R. Diaz-Uriarte, S.A. Alvarez, "Gene Selection and Classification of Microarray Data Using Random Forests”, BMC.Bioinformatics., vol.7, pp.3, 2006.

16. K. Sikonja, “An Adaptation of Relief for attribute Estimation in Regression”, Machine Learning, pp.296-304, 1997.

17. D. Aha, D. Kibler, “Instance-Based Learning Algorithms”, Machine Learning, vol.6, pp.37-66,1996.

18. D. Semitekos, N. Avouris, "Steady State Contingency Analysis of Electrical Networks Using Machine Learning Techniques”, Artificial Intelligence Applications \& Innovations, vol.204, pp. 281-289, 2006.

19. http://en.wikipedia.org/wiki/CDNA_microarray, 2007

20. M.A. Hall, G. Holmes, "Benchmarking Attribute Selection Techniques for Discrete Class Data Mining”, IEEE Trans Knowl Data Eng, vol.15, pp.1437-1447, 2003.

21. J.A. Cruz and D.S. Wishart, “Applications of Machine Learning in Cancer Prediction and Prognosis”, Cancer Informatics, vol.2, pp. 59-78, 2006.

22. D. Berrar, B. Sturgeon, L. Bradbury, W. Dubitzky, "Microarray Data Integration and Machine Learning Techniques for Lung Cancer Survival Prediction”, Critical Assessment of Microarray Data Analysis, pp. 43-54, 2003.

23. M. Shipp, K.N. Ross, P. Tamayo, A.P. Weng, J.L. Kutok, R.C.T. Agular et al., "Diffuse Large B-Cell Lymphoma Outcome Prediction by Gene Expression Profiling and Supervised Machine Learning”, Nature Medicine, vol.8, pp. 68-74, 2002.

24. U.T. Shankavaram, W.C. Reinhold, S. Nishizuka, S. Major, D. Morita, K.K. Chary, "Transcript and Protein Expression Profiles of the NCI-60 Cancer Cell Panel: an Integromic Microarray Study”, Mol Cancer Ther, vol.6, no.3, 2007.

25. M.A. Hall, “Feature Selection for Discrete and Numeric Class Machine Learning”, International Conference on Machine Learning, pp. 359-366, 1999

26. R. Kohavi, “A Study of Cross-Validation and Bootstrap for Accuracy Estimation and 
Model Selection”, Int Jt Conf Artif Intell, pp.1137-1143, 1995

27. Y Ma, Z Ding, Y Qian et al., "Predicting Cancer Drug Response by Proteomic Profiling”, Clinical Cancer Research, vol.12, pp.4583-4589, 2006.

28. R. Rifkin, S. Mukherjee, P. Tamayo et al., "An Analytical Method for Multiclass Molecular Cancer Classification”, SIAMRev, vol.45, pp.706-7 23, 2003.

29. D. Shalon, S.J. Smith, P.O. Brown, “A DNA Microarray System for Analyzing Complex DNA Samples using Two-Color Fluorescent Probe Hybridization”, Genome Res., vol.6, pp.639-645,1996.

30. K.J. Bussey, D. Kane, M. Sunshine, S. Narasimhan, S. Nishizuka, W.C. Reinhold et al., "MatchMiner: a Tool for Batch Navigation among Gene and Gene Product Identifiers”, Genome Biol., vol.4, pp.R27, 2003

31. O. Troyanskaya, M. Cantor, G. Sherlock, P. Brown, T. Hastie, R. Tibshirani et al., "Missing Value Estimation Methods for DNA Microarrays", Bioinformatics., vol.17, pp.520-525,2001.

32. T. Speed et al., Statistical Analysis of Gene Expression Microarray Eata, Chapman \& Hall/CRC, pp.93-159, 2003. 